\title{
Numerical simulation of dynamic fracture using finite elements with embedded discontinuities
}

\author{
Francisco Armero • Christian Linder
}

Received: 16 March 2009 / Accepted: 2 September 2009 / Published online: 18 October 2009

(C) The Author(s) 2009. This article is published with open access at Springerlink.com

\begin{abstract}
This paper presents the extension of some finite elements with embedded strong discontinuities to the fully transient range with the focus on dynamic fracture. Cracks and shear bands are modeled in this setting as discontinuities of the displacement field, the so-called strong discontinuities, propagating through the continuum. These discontinuities are embedded into the finite elements through the proper enhancement of the discrete strain field of the element. General elements, like displacement or assumed strain based elements, can be considered in this framework, capturing sharply the kinematics of the discontinuity for all these cases. The local character of the enhancement (local in the sense of defined at the element level, independently for each element) allows the static condensation of the different local parameters considered in the definition of the displacement jumps. All these features lead to an efficient formulation for the modeling of fracture in solids, very easily incorporated in an existing general finite element code due to its modularity. We investigate in this paper the use of this finite element formulation for the special challenges that the
\end{abstract}

F. Armero $(\varangle) \cdot$ C. Linder

Department of Civil and Environmental Engineering, University of California, Berkeley, CA 94720-1710, USA

e-mail: armero@ce.berkeley.edu

Present Address:

C. Linder

Institute of Applied Mechanics (Chair I), University of Stuttgart, Pfaffenwaldring 7, 70550 Stuttgart, Germany

e-mail: linder@mechbau.uni-stuttgart.de dynamic range leads to. Specifically, we consider the modeling of failure mode transitions in ductile materials and crack branching in brittle solids. To illustrate the performance of the proposed formulation, we present a series of numerical simulations of these cases with detailed comparisons with experimental and other numerical results reported in the literature. We conclude that these finite element methods handle well these dynamic problems, still maintaining the aforementioned features of computational efficiency and modularity.

Keywords Dynamic fracture - Finite elements . Embedded discontinuities - Failure mode transition . Crack branching

\section{Introduction}

The finite element modeling of fracture and failure has been and continues to be an area of intensive research for its importance in many practical applications. A main challenge is the highly non-smooth character of the solutions involved. For instance, we observe the need to deal with discontinuous displacement fields across evolving surfaces of discontinuity, the so-called strong discontinuities, when dealing with propagating cracks. The difficulties increase considerably when accounting for dynamic effects, as it is the focus of this paper. Brittle/ductile failure mode transitions and 
crack branching are phenomena often observed in these conditions.

Different approaches can be found in the literature for the finite element modeling of propagating cracks, shear bands and similar localized failures of solids. For example, the use of cohesive elements across element edges was developed in Needleman (1987), Tvergaard (1990), Xu and Needleman (1994), Camacho and Ortiz (1996), Ortiz and Pandolfi (1999), among others. The dependence of these techniques on the alignment of the mesh can be diminished with the consideration of adaptive refinement techniques, as considered in Ingraffea and Saouma (1985), Ortiz and Quigley (1991), Marusich and Ortiz (1995), Bittencourt et al. (1996), or Carter et al. (2000), to mention just a few.

The complexity of these approaches has motivated the formulation of alternative techniques that do not rely on special alignments of the underlying finite element mesh. The discontinuity then has to be captured in the interiors of the finite elements. Early approaches include the work of Dvorkin et al. (1990) and the general framework for finite elements with embedded strong discontinuities presented in Simo et al. (1993), Armero and Garikipati (1995, 1996), Oliver (1996a,b), and Larsson et al. (1998), to just mention some early publications. Other approaches not sharing this element-based enhancement rely on nodally-based enrichments, like the so-called extended finite element method and its variations as presented in Belytschko and Black (1999), Moës et al. (1999), Dolbow et al. (2000), Wells and Sluys (2001), Wells et al. (2002), or Hansbo and Hansbo (2004), among others. See Oliver et al. (2006) for a comparative study between the local element-based enhanced elements and nodally-enriched formulations, especially in terms of the associated computational costs.

An attractive aspect of the original finite elements with embedded strong discontinuities is the completely local nature of the enhancements required to capture the displacement jumps, local in the sense that they are defined at the element level, independent for each element. This feature arises from a global/local multiscale treatment of these discontinuous solutions as developed in Armero (1999, 2001). In practice, this important characteristic leads not only to a computationally very efficient approach (thanks to the local static condensation of the local degrees of freedom capturing the displacement jumps), but also to a technique that can be easily incorporated into an existing finite element code due to its final modularity. This framework is to be contrasted with the aforementioned extended finite element methods, where additional (global) nodal degrees of freedom, which change as the discontinuity propagates, are introduced.

In this paper we consider specifically the finite elements with embedded discontinuities presented recently in Linder and Armero (2007) in the infinitesimal range, later extended to the finite deformation range in Armero and Linder (2008). In contrast to the original elements with piece-wise constant interpolations of the displacement jumps along the discontinuity and the elements with only linear normal jumps of Manzoli and Shing (2006) in the context of regularized discontinuities, the new elements consider a linear interpolation of both the normal and tangential displacement jumps at the element level. In addition, any underlying finite element can be considered in this framework, including basic displacement-based elements and other formulations treated in general as an assumed strain or B-bar approach. This richer interpolation is crucial to arrive to quadrilateral elements that are able to model the discontinuities without the so-called stress-locking, that is, the spurious transfer of stresses through the discontinuity when modeling a fully softened opening discontinuity. The local enhancement of the element strains is actually obtained with the direct incorporation of the separation mode associated with a linearly opening discontinuity. This approach is to be traced back to the finite elements with embedded softening hinges developed for beams and plates in Ehrlich and Armero (2005) and Armero and Ehrlich (2006a,b). The more complex nature of the kinematics of these structural systems, in terms of deflections and rotations, required the consideration of non-constant generalized displacement jumps.

The goal of this paper is to extend and evaluate the use of the new finite elements for continuum plane problems in the fully dynamic range, with dynamic fracture being at the center of our motivation. The consideration of dynamic effects in finite element formulations with embedded discontinuities is rare; we can only quote Huespe et al. (2006), but within the context of regularized strong discontinuities. As shown in the current paper, this extension is easily achieved after observing that the local problem modeling the strong discontinuity is not directly affected by the dynamic effects. These effects are incorporated through the global response of the solid, a task that can be easily 
accomplished again with standard finite element treatments (e.g typical inertial terms based on a standard global mass matrix). In this way, we preserve the characteristic features of the considered approach noted above, namely, its completely local nature leading to a computationally very efficient formulation, easily incorporated in an existing finite element code due to its modularity. The numerical results reported in this paper show also the appropriateness of the approach in modeling different challenging situations in dynamic fracture, namely, failure mode transitions and crack branching.

An outline of the rest of the paper is as follows. Section 2 describes the standard mechanical initial boundary value problem and its finite element approximation. This problem defines the aforementioned (global) large scale, where we use standard finite element methods. The strong discontinuities are introduced in the small scales defined by the finite elements as discussed in Sect. 3. To emphasize the overall structure of the considered formulation, we discuss briefly its numerical implementation in Sect. 4, leaving the design of the enhanced operators capturing a linearly opening discontinuity in the Appendix. Section 5 presents the results obtained with the proposed formulation for several benchmark problems, including detailed comparisons with experimental tests and other numerical results reported in the literature. Finally, Sect. 6 concludes with a brief summary of the main features of the finite element methods considered in the paper and an outlook on our current and future work in this area.

\section{The mechanical initial boundary value problem}

This section summarizes the standard mechanical initial boundary value problem. The continuum problem is presented in Sect. 2.1 with its discrete approximation outlined in Sect. 2.2. The strong discontinuities employed to model the failure of the solid will be introduced in this framework in Sect. 3.

\subsection{The continuum problem}

We consider a solid body represented by a fixed open domain $\Omega \subset \mathbb{R}^{n_{\mathrm{dim}}}$ for $1 \leq n_{\mathrm{dim}} \leq 3$ under the standard assumption of infinitesimal deformations described by a displacement field $\boldsymbol{u}: \Omega \times[0, T] \rightarrow \mathbb{R}^{n_{\mathrm{dim}}}$ in time $t \in[0, T] \subset \mathbb{R}_{+}$for some time interval $T>0$. The solid's material particles are labeled by their positions $\boldsymbol{x} \in \Omega$, and the solid's density is denoted by $\rho(\boldsymbol{x}, t)$. In this context, we consider the associated infinitesimal strain tensor $\varepsilon: \Omega \times[0, T] \rightarrow \mathbb{R}_{\text {sym }}^{n_{\operatorname{dim}} \times n_{\operatorname{dim}}}$ (the space of symmetric tensors) defined by

$\boldsymbol{\varepsilon}(\boldsymbol{u})=\nabla^{s} \boldsymbol{u}=\frac{1}{2}\left[\nabla \boldsymbol{u}+(\nabla \boldsymbol{u})^{T}\right]$

for the standard gradient operator $\nabla$ in $\boldsymbol{x}$. Similarly, we denote by

$\boldsymbol{v}(\boldsymbol{x}, t)=\frac{\partial}{\partial t} \boldsymbol{u}(\boldsymbol{x}, t)(\equiv \dot{\boldsymbol{u}})$

and

$\boldsymbol{a}(\boldsymbol{x}, t)=\frac{\partial}{\partial t} \boldsymbol{v}(\boldsymbol{x}, t)=\frac{\partial^{2}}{\partial t^{2}} \boldsymbol{u}(\boldsymbol{x}, t)(\equiv \ddot{\boldsymbol{u}})$

the associated velocity and acceleration fields. We denote the stresses in the solid by $\sigma: \Omega \times[0, T] \rightarrow$ $\mathbb{R}_{\text {sym }}^{n_{\text {dim }} \times n_{\text {dim }}}$ (a symmetric tensor under the usual argument of balance of angular momentum), given in terms of the strains $\varepsilon$ through a constitutive relation for the material response as described in Sect. 3 below.

If the solid is subjected to a specific volumetric body force $\boldsymbol{b}: \Omega \rightarrow \mathbb{R}^{n_{\mathrm{dim}}}$ (per unit mass) and imposed boundary tractions $\overline{\boldsymbol{t}}: \partial_{t} \Omega \rightarrow \mathbb{R}^{n_{\mathrm{dim}}}$ for some part of the boundary $\partial_{t} \Omega \subset \partial \Omega$, the balance of linear momentum can be written in weak form as

$$
\begin{gathered}
\int_{\Omega} \rho \ddot{\boldsymbol{u}} \cdot \delta \boldsymbol{u} d \Omega+\int_{\Omega} \boldsymbol{\sigma}: \nabla^{s}(\delta \boldsymbol{u}) d \Omega \\
=\int_{\Omega} \rho \boldsymbol{b} \cdot \delta \boldsymbol{u} d \Omega+\int_{\partial_{t} \Omega} \overline{\boldsymbol{t}} \cdot \delta \boldsymbol{u} \mathrm{d} \Gamma
\end{gathered}
$$

for all admissible variations $\delta \boldsymbol{u}$, that is, $\delta \boldsymbol{u}=0$ on $\partial_{u} \Omega \subset \partial \Omega$, the part of the boundary where the displacement field is imposed as $\boldsymbol{u}=\overline{\boldsymbol{u}}$ for some given function $\overline{\boldsymbol{u}}: \partial_{u} \Omega \rightarrow \mathbb{R}^{n_{\mathrm{dim}}}$. We assume the standard conditions of non-overlapping parts $\partial_{u} \Omega$ and $\partial_{t} \Omega$ in each component of the displacements and traction, covering all the boundary $\partial \Omega$ for a well-posed problem. The secondorder in time problem (4) in the displacement $\boldsymbol{u}(\boldsymbol{x}, t)$ is supplemented by initial conditions on the displacement $\boldsymbol{u}(\boldsymbol{x}, 0)=\boldsymbol{u}_{0}(\boldsymbol{x})$ and velocity $\boldsymbol{v}(\boldsymbol{x}, 0)=\boldsymbol{v}_{0}(\boldsymbol{x})$ in the dynamic range of interest here.

\subsection{The finite element framework}

The continuum problem summarized in the previous section corresponds to the standard initial boundary value problem in solid mechanics in the global setting of the domain $\Omega$, involving in particular standard 
regularity conditions for the different fields involved (e.g. $H^{1}(\Omega)$ functions for the displacements as in typical treatments). Additional considerations involving the so-called strong discontinuities (discontinuities in the displacement field) are to be accounted for locally, instead of globally, as discussed in the following section. This standard character of the global problem considered so far emphasizes our intention to employ standard finite element methods at this level.

In this way, we proceed with standard considerations and introduce a finite element approximation of the displacement field

$\boldsymbol{u}(\boldsymbol{x}, t) \approx \boldsymbol{u}^{h}\left(\boldsymbol{x}^{h}, t\right)=\sum_{A=1}^{n_{\text {node }}} N^{A}\left(\boldsymbol{x}^{h}\right) \mathbf{d}_{A}(t)=\mathbf{N d}$

for $x^{h} \in \Omega^{h}$ in terms of a standard set of shape functions $N^{A}$ and a set of discrete (nodal) displacements $\mathbf{d}_{A}(t)$, usually associated to a set of nodes $A=1, \ldots, n_{\text {node }}$ of a finite element mesh $\Omega^{h}=\cup_{e=1}^{n_{\text {elem }}} \Omega_{e}^{h}$ covering the domain $\Omega$ with $n_{\text {elem }}$ elements $\Omega_{e}^{h}$. The same finite element interpolations are assumed to define the velocity $\boldsymbol{v}^{h}\left(\boldsymbol{x}^{h}, t\right)$ and acceleration $\boldsymbol{a}^{h}\left(\boldsymbol{x}^{h}, t\right)$ in terms of nodal velocities $\mathbf{v}_{A}(t)$ and nodal accelerations $\mathbf{a}_{A}(t)$, respectively, for $A=1, \ldots, n_{\text {node }}$. We denote by $\mathbf{d}, \mathbf{v}$, and a the corresponding global arrays, all three understood as time functions, and the corresponding global array $\mathbf{N}$ of (fixed in time) shape functions.

We have also denoted by $\boldsymbol{x}^{h} \in \Omega^{h}$ the position coordinates in the discrete geometry, defined as usual through the isoparametric concept by the same relation (5) but in terms of the nodal coordinates $\mathbf{x}_{A}$, globally assembled in the vector $\mathbf{x}$.

To accommodate any finite element treatment of the continuum problem at hand, we adopt a general assumed strain formulation by which the strain field is approximated as

$\boldsymbol{\varepsilon}(\boldsymbol{u}(\boldsymbol{x}, t)) \approx \boldsymbol{\varepsilon}^{h}(\mathbf{d})=\sum_{A=1}^{n_{\text {node }}} \overline{\boldsymbol{B}}^{A}\left(\boldsymbol{x}^{h}\right) \boldsymbol{d}_{A}(t)=\overline{\mathbf{B}} \mathbf{d}$

for $\boldsymbol{x}^{h} \in \Omega^{h}$ in terms of an assumed strain operator $\overline{\mathbf{B}}\left(\boldsymbol{x}^{h}\right)$ (Hughes 1987). Similarly, we write

$\nabla^{s}(\delta \boldsymbol{u}) \approx \overline{\mathbf{B}} \delta \mathbf{d}$

for the nodal displacement variations $\delta \mathbf{d}$. Inserting these relations into the governing Eq. (4) and following standard arguments in finite element analysis, we arrive at the (global) discrete system of equations
$\mathbf{R}=\mathbf{f}_{e x t}-\boldsymbol{A}_{e=1}^{n_{\text {elem }}}\left(\int_{\Omega_{e}^{h}} \overline{\mathbf{B}}^{T} \boldsymbol{\sigma} \mathrm{d} \Omega\right)-\mathbf{M a}=0$

where we have introduced the external force vector

$\mathbf{f}_{e x t}=\int_{\Omega^{h}} \mathbf{N}^{T} \rho \mathbf{b} \mathrm{d} \Omega+\int_{\partial_{t} \Omega^{h}} \mathbf{N}^{T} \overline{\mathbf{t}} \mathrm{d} \Gamma$

the assembly operator $\mathbf{A}$ for the $n_{\text {elem }}$ element contributions and the mass matrix

$\mathbf{M}=\bigoplus_{e=1}^{n_{\text {elem }}} \mathbf{M}^{e} \quad$ for $\quad \mathbf{M}^{e}=\int_{\Omega_{e}^{h}} \rho \mathbf{N}^{T} \mathbf{N} \mathrm{d} \Omega$

or any alternative assumed expression like a lumped mass, capturing the dynamic effects. We refer to e.g. Hughes (1987) for complete details in all these considerations.

To summarize the developments in this section, we have considered the standard initial boundary value problem describing the deformation of a solid in the dynamic range and its standard finite element approximation. Our purpose in elaborating on some of the details is to emphasize the standard character of these considerations, besides defining the notation to be used in the following sections. We note again the goal to incorporate the strong discontinuities characterizing the failure of the solid without breaking this standard structure of the problem at hand and its numerical approximation.

\section{The incorporation of the strong discontinuities}

Two related issues remain to be addressed in the context of the mechanical problem described in the previous section. First, we have to introduce the constitutive relation defining the stress $\sigma$ in terms of the strain $\boldsymbol{\varepsilon}$ and any needed internal variables characterizing the response of the material. The second issue, and main goal in the developments of this paper, is the consideration of discontinuities of the displacements (the strong discontinuities) modeling the localized failure of the material in the form of cracks and shear bands, for example, in both the continuum and finite element frameworks described above. In particular, a key aspect of this goal is to sharply resolve the kinematics of these discontinuous solutions by local considerations associated to the 
small scales of the material response, maintaining the structure of those global or large-scale frameworks.

These arguments lead naturally to the consideration of a multi-scale treatment of the problem at hand, originally presented in Armero $(1999,2001)$ and briefly summarized in Sect. 3.1. This point of view leads naturally to the resolution of the strong discontinuities through local enhancements of the finite elements in the discrete framework as discussed in Sect. 3.2, summarizing the developments of new finite elements presented recently in Linder and Armero (2007) for the static infinitesimal case and, in this way, extending their use and evaluation to the dynamic range of interest in the current paper.

\subsection{The continuum local problem with strong discontinuities}

We identify the small scales of the material response at a material point $x \in \Omega$ with a local neighborhood $\Omega_{x} \subset$ $\Omega$ of $\boldsymbol{x}$. In this context, we consider a strong discontinuity characterized by a surface $\Gamma_{x} \subset \mathbb{R}^{n_{\mathrm{dim}}-1}$ with unit normal $\mathbf{n}$. The appearance of this surface is detected by a physical condition based on the bulk response of the material in $\Omega_{x}$. In general, we consider the loss of ellipticity condition in terms of the acoustic tensor of the tangent response of the material in the bulk (see Armero and Garikipati 1995, 1996), particularizing for example to the standard maximum normal stress criterion for brittle failures.

The kinematics of the deformation in this small scale is assumed characterized by a local displacement field $\boldsymbol{u}_{\mu}$ which can always be written as

$\boldsymbol{u}_{\mu}=\boldsymbol{u}+\tilde{\boldsymbol{u}}\left(\llbracket \boldsymbol{u}_{\mu} \rrbracket\right)$ in $\Omega_{x}$

in terms of the original global displacement field $\boldsymbol{u}$ in $\Omega$ and an added part part $\tilde{\boldsymbol{u}}\left(\llbracket \boldsymbol{u}_{\mu} \rrbracket\right)$ expressed as a function of the displacement jumps $\llbracket \boldsymbol{u}_{\mu} \rrbracket: \Gamma_{x} \rightarrow \mathbb{R}^{n_{\text {dim }}}$ associated to the strong discontinuity. We refer to Fig. 1 for an illustration of these considerations.

The small-scale displacements (11) define the local strain

$\boldsymbol{\varepsilon}_{\mu}=\boldsymbol{\varepsilon}(\boldsymbol{u})+\tilde{\boldsymbol{\varepsilon}}\left(\llbracket \boldsymbol{u}_{\mu} \rrbracket\right)$ in $\Omega_{x} \backslash \Gamma_{x}$

for the large-scale strain $\boldsymbol{\varepsilon}(\boldsymbol{u})$ defined in (1) with an additional contribution depending on the added field $\llbracket \boldsymbol{u}_{\mu} \rrbracket$. Here we consider the bulk $\Omega_{x} \backslash \Gamma_{x}$ of the smallscale neighborhood $\Omega_{x}$ where the small-scale displacement defines a regular strain field. We can think of the

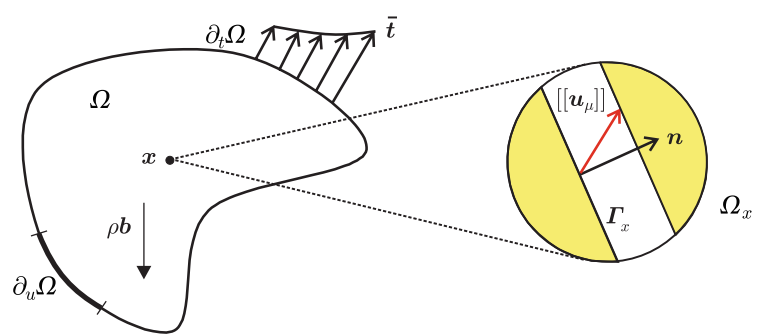

Fig. 1 Illustration of the continuum framework of the local problem, where the global problem is defined in the body $\Omega$, including the applied loading and imposed boundary conditions, with the strong discontinuity appearing in the small scales of a local neighborhood $\Omega_{x}$ of a material point $x$

discontinuous part defining a singular distribution on the surface $\Gamma_{x}$ (i.e. a Dirac delta function).

Important to the considerations here is that the regular strain field $\boldsymbol{\varepsilon}_{\mu}$ in (12) characterizes the bulk response of the material and defines the stress field $\sigma$ through a standard constitutive material model. For example, the cases presented in Sect. 5 consider a $J_{2}$-theory plasticity model for a ductile material (steel) and a basic linear elastic response for a brittle material (PMMA); see Sect. 5 for complete details.

Besides this bulk response, the full characterization of the material behavior requires a constitutive description of the strong discontinuity $\Gamma_{x}$ itself. This corresponds to a cohesive law between the so-called driving traction $\mathbf{t}_{\Gamma}$ and the displacement jumps $\llbracket \boldsymbol{u}_{\mu} \rrbracket$, allowing the accommodation of general effects like damage, plastic, poroplastic, etc. responses $\mathbf{t}_{\Gamma}\left(\llbracket \boldsymbol{u}_{\mu} \rrbracket\right)$ as needed for the particular problem at hand.

The introduction of the local field $\llbracket \boldsymbol{u}_{\mu} \rrbracket$ on $\Gamma_{x}$ requires the consideration of an additional equation closing the formulation. This is accomplished by defining the driving traction as the trace of the stress field on $\Gamma_{x}$, a relation we refer as local equilibrium. In weak form, we can write

$\int_{\Gamma_{x}} \delta \llbracket \boldsymbol{u}_{\mu} \rrbracket \cdot\left(\boldsymbol{\sigma} \boldsymbol{n}-\mathbf{t}_{\Gamma}\right) \mathrm{d} \Gamma=0$

for all variations $\delta \llbracket \boldsymbol{u}_{\mu} \rrbracket: \Gamma_{x} \rightarrow \mathbb{R}^{n_{\mathrm{dim}}}$ of the displacement jumps. For the dynamic context of interest in this paper, we can observe that implicit in this equation is that no mass is associated to the discontinuity surface $\Gamma_{x}$, as one would expect from physical considerations of the problem at hand. The mass is associated with the bulk of the material. 
Important to the considerations here is the local character of Eq. (13), holding in $\Omega_{x}$ (actually $\Gamma_{x} \subset \Omega_{x}$ ) and not the global domain $\Omega$. In fact, as shown in Armero (2001), the problem thus defined allows to explicitly solve for the local field $\llbracket \boldsymbol{u}_{\mu} \rrbracket$ in terms of the large-scale displacement $\boldsymbol{u}$ in the large-scale limit

$$
\begin{aligned}
h_{x}:=\frac{A_{x}}{l_{\Gamma_{x}}} & \rightarrow 0 \text { for } A_{x}=\int_{\Omega_{x}} \mathrm{~d} \Omega \text { and } \\
l_{\Gamma_{x}} & =\int_{\Gamma_{x}} \mathrm{~d} \Gamma
\end{aligned}
$$

that is, of vanishing small scales $\Omega_{x}$, the case of interest here.

\subsection{The discrete local problem with strong discontinuities}

The considerations presented in the previous section for the introduction of the strong discontinuities through a local neighborhood of the material points in the continuum fit exactly the framework of a finite element method by identifying this neighborhood with a finite element. Note in this respect the interest of considering the large-scale limit, as expressed by the relation (14), of vanishing finite elements in the limit of fine meshes. The main goal is then to define the proper interpolation of the added discontinuity fields and equations so that an accurate and sharp resolution of their effects is captured by the finite element.

The implementation used in the plane examples presented in Sect. 5 represents the discontinuity line by piece-wise straight segments propagating through the mesh. A particular element captures one of these segments, once the propagation condition alluded to above is fulfilled by the state of stress in the element, defining in the process the orientation and location of the segment $\Gamma_{e}^{h}$. We refer to Sect. 5 below for additional details in each of the physical problems considered in the numerical simulations presented in that section.

Our starting situation here is then a finite element crossed by a discontinuity segment, which is defined by a unit normal $\boldsymbol{n}$ and mid-point $\boldsymbol{x}_{\Gamma_{e}^{h}}^{h}$ as sketched in Fig. 2. In this setting we introduce the coordinate $s$ along $\Gamma_{e}^{h}$ from $x_{\Gamma_{e}^{h}}^{h}$ and a general interpolation of the displacement jumps

$\llbracket \boldsymbol{u}_{\mu} \rrbracket(s) \approx \llbracket \boldsymbol{u}_{\mu}^{h} \rrbracket(s)=\mathbf{D}(s) \boldsymbol{\xi}$

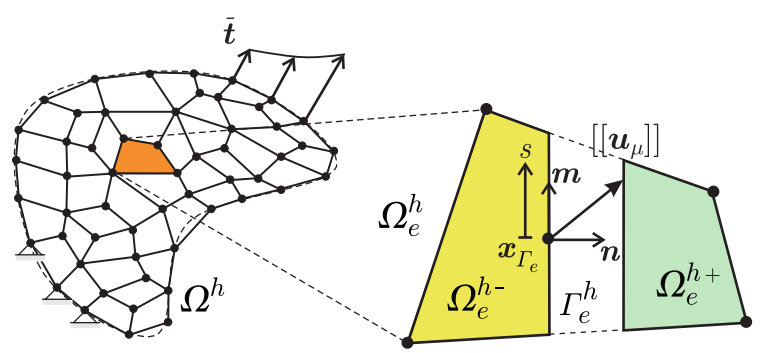

Fig. 2 Illustration of the discrete description of the local problem, where the global problem is defined by a mesh $\Omega^{h}$ covering the body $\Omega$, and the applied external loading and imposed boundary conditions. The strong discontinuity appears in the small scales of a local neighborhood $\Omega_{e}^{h}$ corresponding to a single finite element in the discrete framework

for a set of local element parameters $\xi$ and associated jump interpolation functions $\mathbf{D}(s)$. For example, we are interested in the piece-wise linear interpolation

$\llbracket \boldsymbol{u}_{\mu}^{h} \rrbracket(s)=\xi_{\langle 0 n\rangle} \boldsymbol{n}+\xi_{\langle 0 m\rangle} \boldsymbol{m}+s \xi_{\langle 1 n\rangle} \boldsymbol{n}+s \xi_{\langle 1 m\rangle} \boldsymbol{m}$

So

$\mathbf{D}(s)=\left[\begin{array}{llll}\boldsymbol{n} & \boldsymbol{m} & s \boldsymbol{n} & s \boldsymbol{m}\end{array}\right]$

in terms of a total of four local parameters $\xi$ for the normal and tangential components of the displacement jumps. This interpolation improves on the earlier and simpler piece-wise constant interpolation considering only the constant part of (16), especially for quadrilateral elements; see Linder and Armero (2007) for details. We emphasize again the local character of the parameters $\xi$, local in the sense that they are defined for each finite element independently of the rest once the corresponding discontinuity segment is activated.

With these considerations at hand, we write the local equilibrium Eq. (13) as

$\mathbf{r}_{\mathrm{enh}}^{e}=-\int_{\Omega_{e}^{h}} \mathbf{G}_{(e)}^{T} \boldsymbol{\sigma} \mathrm{d} \Omega-\int_{\Gamma_{e}^{h}} \mathbf{D}^{T} \mathbf{t}_{\Gamma} \mathrm{d} \Gamma=0$

for $\Omega_{e}^{h} \in \mathcal{E}_{\text {disc }}$, where $\mathcal{E}_{\text {disc }}$ denotes the set of elements $\Omega_{e}^{h}$ with such an activated discontinuity segment. The minus sign in the definition of the local residual $\mathbf{r}_{\mathrm{enh}}^{e}$ in (18) is just for convenience in the numerical implementation, as presented in Sect. 4. The local character of equation (18), holding independently for each element $\Omega_{e}^{h} \in \mathcal{E}_{\text {disc }}$ with its associated segment $\Gamma_{e}^{h}$, is a result again of the assumed piece-wise continuous interpolation for the displacement jumps and it is heavily exploited in an efficient numerical implementation of the final finite element method, as discussed in Sect. 4 below. 
We have introduced the element operator $\mathbf{G}_{(e)}$ in Eq. (18). Comparing this equation with the original Eq. (13), this operator must define the tractions on $\Gamma_{e}^{h}$ associated with the stresses $\sigma$ in the element. This choice in writing Eq. (18) is motivated by the definition of these stresses at the quadrature points used in the evaluation of integrals like (8) in the bulk of the element $\Omega_{e}^{h}$ in a typical finite element formulation. Indeed, the use of this operator implies that all integrals in the discrete governing Eqs. (8) and (18) can be evaluated by standard quadrature rules

$$
\begin{aligned}
\int_{\Omega_{e}^{h}}(\cdot) \mathrm{d} \Omega & =\sum_{l=1}^{l_{\mathrm{int}}^{\Omega}}(\cdot)_{l} w_{l}^{\Omega} j_{l} \quad \text { and } \\
\int_{\Gamma_{e}^{h}}(\cdot) \mathrm{d} \Gamma & =\sum_{l=1}^{l_{\mathrm{int}}^{\Gamma}}(\cdot)_{l} w_{l}^{\Gamma} l_{\Gamma_{e}^{h}}
\end{aligned}
$$

on the element $\Omega_{e}^{h}$ and along the strong discontinuity segment $\Gamma_{e}^{h}$. We have denoted by $l_{\text {int }}^{\Omega}$ and $l_{\text {int }}^{\Gamma}$ the number of integration points and by $w_{l}^{\Omega}$ and $w_{l}^{\Gamma}$ the integration weights of the respective quadratures, the latter normalized by the pointwise Jacobian $j_{l}$ and the discontinuity length $l_{\Gamma_{e}^{h}}$, respectively. Hence, as illustrated in Fig. 3 the operator $\mathbf{G}_{(e)}$ can be thought as a projection operator taking the stresses at the bulk quadrature points to the associated traction along $\Gamma_{e}^{h}$ and, as discussed in the Appendix, it can be defined in closed-form once the geometry of the crossing segment $\Gamma_{e}^{h}$ is determined for any given element $\Omega_{e}^{h}$.

The evaluation of the discrete Eqs. (8) and (18) is completed with the use of the constitutive equations for the bulk stresses $\sigma$ and the driving traction $\mathbf{t}_{\Gamma}$. The latter is given in terms of the displacement jumps (16) along

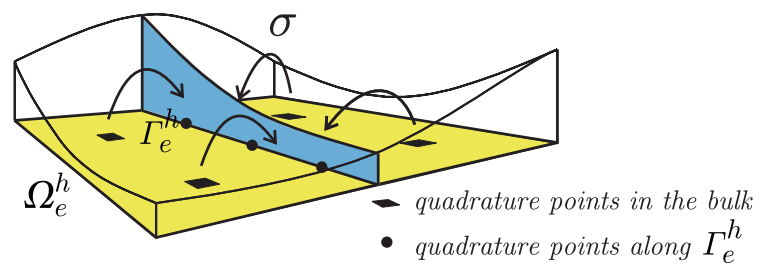

Fig. 3 Illustration of the equilibrium operator $\mathbf{G}_{(e)}$ for a quadrilateral element. The stresses $\sigma$ are defined at the quadrature points in the bulk of the element $\Omega_{e}^{h}$ whereas the driving traction $\mathbf{t}_{\Gamma}$ is naturally defined at the quadrature points associated to the strong discontinuity $\Gamma_{e}^{h}$. The equilibrium operator $\mathbf{G}_{(e)}$ recovers the traction associated with the bulk stresses on the discontinuity $\Gamma_{e}^{h}$ the discontinuity segment $\Gamma_{e}^{h}$. As discussed in the previous section, the stresses, on the other hand, are given in terms of the small-scale strains (12) corresponding to an additive enhancement of the large-scale strains. Hence, in the finite element framework of interest here, we consider

$\boldsymbol{\varepsilon}_{\mu}^{h}=\overline{\mathbf{B}} \mathbf{d}+\mathbf{G}_{(c)} \boldsymbol{\xi} \quad$ in $\quad \Omega_{e}^{h} \backslash \Gamma_{e}^{h}$

for an enhanced strain operator $\mathbf{G}_{(c)}$ different, in general, to the previous equilibrium operator $\mathbf{G}_{(e)}$. The subscript "c" in this new operator stands for compatibility, since it refers to the strains. The linearity of the enhanced strains (20) in the displacement jump parameters $\boldsymbol{\xi}$ follows from the assumed infinitesimal range of deformations (as the large-scale strains $\overline{\mathbf{B}} \mathbf{d})$.

We can observe that the enhanced operator $\mathbf{G}_{(c)}$ must capture the kinematics of a separating discontinuity in the context defined by the discrete kinematics of the underlying finite element. In particular, it must be able to capture the separation mode depicted in Fig. 4 for the linear displacement jumps (16). The mode consists of one side of the element across the discontinuity segment $\Gamma_{e}^{h}$ separating and infinitesimally rotating while uniformly stretching in the tangential direction to the discontinuity. No other strains must appear, hence avoiding the appearance of spurious stresses transferring across the discontinuity, the so-called stress locking. This observation leads naturally to a simple procedure for the construction of the operator $\mathbf{G}_{(c)}$ as summarized in the Appendix. Remarkably, the final

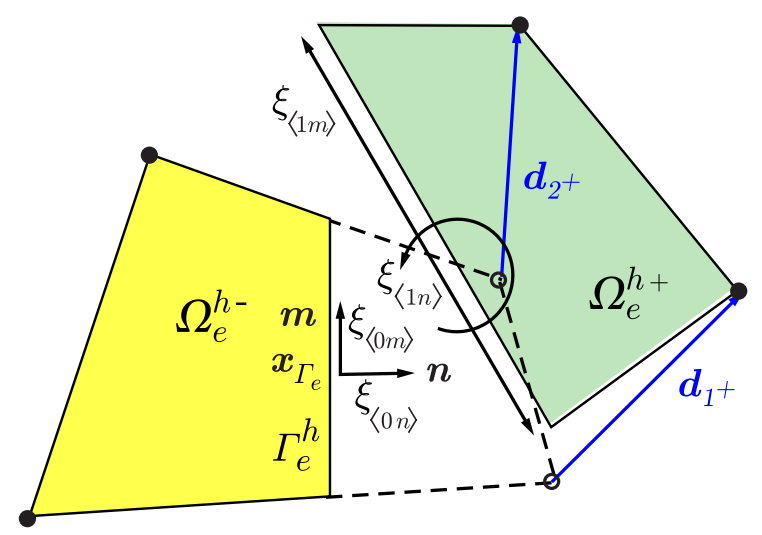

Fig. 4 Illustration of the linear separation mode to be captured with the finite elements with embedded discontinuities. It consists of one side of the element separating and (infinitesimally) rotating while stretching in the tangential direction to the discontinuity segment $\Gamma_{e}^{h}$ 
numerical formulation does not require the consideration of a local discontinuous displacement field in the element $\Omega_{e}^{h} \in \mathcal{E}_{d i s c}$, as considered in (11) for the local neighborhood $\Omega_{x}$ in the continuum.

\section{Numerical implementation}

The previous section outlined the finite element formulation with embedded strong discontinuities considered in this work. With the purpose of emphasizing the efficiency of the formulation and the simplicity of its implementation in a standard finite element code, we discuss in this section its numerical implementation for the case of interest here, namely, the fully transient case.

The discussion focuses on the solution of the resulting nonlinear enhanced finite element equations. As discussed in the previous section, these equations are constructed once the strong discontinuity is propagated through the element. The first step in this propagation is the activation of the discontinuity segment in a given element through a physical criterion on the stresses at the element, as discussed in Sect. 5 for the different numerical examples presented in that section, defining in the process the orientation of the local segment. A successful activation is obtained by the actual propagation from neighboring elements, with the final position of the segment defined by that orientation and the imposition of the continuity of the path; see Sect. 5.2.2 for variations of this strategy to capture crack branching. All these arguments define an algorithm fully geometrical in nature, carried out after the convergence of a given time step in the incremental/iterative solution procedure discussed next, and for as many elements where an active discontinuity has been detected.

\subsection{The dynamic discrete governing equations and their linearization}

The finite element equations discussed in the previous sections, in particular the residual Eqs. (8) and (18), still need to be discretized in time. We consider a typical time-stepping strategy for a sequence of time intervals $[0, T]=\bigcup_{n}\left[t_{n}, t_{n+1}\right]$ with $\Delta t=t_{n+1}-t_{n}$, not necessarily constant. The numerical simulations presented in Sect. 5 consider in particular a classical Newmark scheme with the residual equations imposed at the end of the time step $t_{n+1}$ as

$$
\begin{aligned}
& \mathbf{R}\left(\mathbf{d}_{n+1}, \boldsymbol{\xi}_{n+1}\right) \\
& =\mathbf{f}_{e t_{n+1}}-\boldsymbol{\Lambda}_{e=1}^{n_{\text {elem }}}\left(\int_{\Omega_{e}^{h}} \overline{\mathbf{B}}^{T} \boldsymbol{\sigma}_{n+1} \mathrm{~d} \Omega\right)-\mathbf{M a}_{n+1} \\
& \mathbf{r}_{\mathrm{enh}}^{e}\left(\mathbf{d}_{n+1_{e}}, \boldsymbol{\xi}_{n+1_{e}}\right)=-\int_{\Omega_{e}^{h}} \mathbf{G}_{(e)}^{T} \boldsymbol{\sigma}_{n+1} d \Omega \\
& -\int_{\Gamma_{e}^{h}} \mathbf{D}^{T} \mathbf{t}_{\Gamma n+1} \mathrm{~d} \Gamma \text { for } \Omega_{e}^{h} \in \mathcal{E}_{\text {disc }_{n}}
\end{aligned}
$$

together with the dynamic update equations

$$
\left.\begin{array}{rl}
\mathbf{d}_{n+1}= & \mathbf{d}_{n}+\Delta t \mathbf{v}_{n}+\frac{(\Delta t)^{2}}{2}\left[(1-2 \beta) \mathbf{a}_{n}\right. \\
& \left.+2 \beta \mathbf{a}_{n+1}\right] \\
\mathbf{v}_{n+1}= & \mathbf{v}_{n}+\Delta t\left[(1-\gamma) \mathbf{a}_{n}+\gamma \mathbf{a}_{n+1}\right]
\end{array}\right\}
$$

in terms of the algorithmic parameters $\beta$ and $\gamma$, for the nodal displacements, velocities and accelerations $(\cdot)_{n+1} \approx(\cdot)\left(t_{n+1}\right)$ at the end of the time increment $t_{n+1}$ in terms of their corresponding values $(\cdot)_{n}$ at $t_{n}$. See Hughes (1987), among many others, for complete details. We have denoted by $\boldsymbol{\sigma}_{n+1}=\boldsymbol{\sigma}\left(\mathbf{d}_{n+1_{e}}, \boldsymbol{\xi}_{n+1_{e}}\right)$ and $\mathbf{t}_{\Gamma n+1}=\mathbf{t}_{\Gamma}\left(\boldsymbol{\xi}_{n+1_{e}}\right)$ for the nodal displacements $\mathbf{d}_{n+1_{e}}$ and enhanced parameters $\boldsymbol{\xi}_{n+1_{e}}$, the latter in the elements in $\mathcal{E}_{d i s c_{n}}$ with an active strong discontinuity. As noted above, the propagation of the discontinuity is carried out at the end of the time step, hence keeping the set of elements $\mathcal{E}_{\text {disc }}$ fixed at $t_{n}$ during the solution of the governing equations in a given time step.

We consider a Newton-Raphson scheme for the solution of the nonlinear algebraic system of Eqs. (21-22). In this setting, these equations are linearized about the last known equilibrium position $\left\{\mathbf{d}_{n+1}^{k}, \boldsymbol{\xi}_{n+1}^{k}\right\}$. Linearization of the residuals $\mathbf{R}$ and $\mathbf{r}_{\text {enh }}^{e}$ with respect to their dependent variables $\mathbf{d}^{k}$ and $\xi^{k}$ results in the stiffness matrix contributions $\mathbf{K}_{d d}^{e^{k}}, \mathbf{K}_{d \xi}^{e^{k}}$, $\mathbf{K}_{\xi d}^{e^{k}}$, and $\mathbf{K}_{\xi \xi}^{e^{k}}$, respectively. The system of linearized equations can then be written as

$$
\left.\begin{array}{l}
\boldsymbol{A}_{e=1}^{n_{\text {elem }}}\left[\widetilde{\mathbf{K}}_{d d}^{e^{k}} \Delta \mathbf{d}_{n+1_{e}}^{k+1}+\mathbf{K}_{d \xi}^{e^{k}} \Delta \xi_{n+1_{e}}^{k+1}\right]=\boldsymbol{\Lambda}_{e=1}^{n_{\text {elem }}} \mathbf{R}^{e^{k}} \\
\mathbf{K}_{\xi d}^{e^{k}} \Delta \mathbf{d}_{n+1_{e}}^{k+1}+\mathbf{K}_{\xi \xi}^{e^{k}} \Delta \xi_{n+1_{e}}^{k+1}=\mathbf{r}_{\mathrm{enh}}^{e^{k}} \\
\text { for } \Omega_{e}^{h} \in \mathcal{E}_{d i s c_{n}}
\end{array}\right\}
$$

for the increments of the element arrays $\Delta \mathbf{d}_{n+1_{e}}$ and $\Delta \boldsymbol{\xi}_{n+1_{e}}$ with the global increments

$\mathbf{d}_{n+1}^{k+1}=\mathbf{d}_{n+1}^{k}+\Delta \mathbf{d}_{n+1}^{k+1}$

and the local (element) updates

$\boldsymbol{\xi}_{n+1_{e}}^{k+1}=\boldsymbol{\xi}_{n+1_{e}}^{k}+\Delta \boldsymbol{\xi}_{n+1_{e}}^{k+1}$ for $\Omega_{e}^{h} \in \mathcal{E}_{\text {disc }_{n}}$ 
with an iteration index $k$.

The different arrays in (23) are evaluated at the last iteration values $\left\{\mathbf{d}_{n+1}^{k}, \xi_{n+1}^{k}\right\}$ using expressions (21) for the residuals, with the tangent stiffness matrices given as

$$
\begin{aligned}
\mathbf{K}_{d d}^{e} & =\int_{\Omega_{e}^{h}} \overline{\mathbf{B}}^{T} \mathbb{C} \overline{\mathbf{B}} \mathrm{d} \Omega \\
\mathbf{K}_{d \xi}^{e} & =\int_{\Omega_{e}^{h}} \overline{\mathbf{B}}^{T} \mathbb{C} \mathbf{G}_{(c)} \mathrm{d} \Omega \\
\mathbf{K}_{\xi d}^{e} & =\int_{\Omega_{e}^{h}} \mathbf{G}_{(e)}^{T} \mathbb{C} \overline{\mathbf{B}} \mathrm{d} \Omega
\end{aligned}
$$

and

$$
\mathbf{K}_{\xi \xi}^{e}=\int_{\Omega_{e}^{h}} \mathbf{G}_{(e)}^{T} \mathbb{C} \mathbf{G}_{(c)} d \Omega+\int_{\Gamma_{e}^{h}} \mathbf{D}^{T} \mathbb{C}_{\Gamma} \mathbf{D} \mathrm{d} \Gamma
$$

where we have used the material tangents $\mathbb{C}$ and $\mathbb{C}_{\Gamma}$ defined by the linearization of the bulk and localized (cohesive law) constitutive relations

$$
\Delta \boldsymbol{\sigma}=\mathbb{C} \Delta \boldsymbol{\varepsilon}_{\mu}^{h} \quad \text { and } \quad \Delta \mathbf{t}_{\Gamma}=\mathbb{C}_{\Gamma} \Delta\left(\llbracket \boldsymbol{u}_{\mu}^{h} \rrbracket\right)
$$

in $\Omega_{e}^{h}$ and along $\Gamma_{e}^{h}$, respectively. We also used the notation

$\widetilde{\mathbf{K}}_{d d}^{e^{k}}=\mathbf{K}_{d d}^{e^{k}}+\frac{1}{\beta(\Delta t)^{2}} \mathbf{M}^{e}$

in (23), accounting for the acceleration and mass term in $(21)_{1}$.

\subsection{Static condensation}

One of the main features of the proposed finite element framework of strong discontinuities is the ability to statically condense out the local element parameters on the element level, leaving the focus again on the solution of the global problem in terms of the global displacement field d. Given the second equation in (23), the increments of the enhanced parameter can be computed in terms of the small-scale residual $\mathbf{r}_{\mathrm{enh}}^{\mathbf{e}^{k}}$ and the displacement increments $\Delta \mathbf{d}_{e}^{k+1}$ as

$\Delta \xi_{n+1_{e}}^{k+1}=\left(\mathbf{K}_{\xi \xi}^{e^{k}}\right)^{-1}\left[\mathbf{r}_{\mathrm{enh}}^{e^{k}}-\mathbf{K}_{\xi d}^{e^{k}} \Delta \mathbf{d}_{n+1_{e}}^{k+1}\right]$

locally for the elements $\Omega_{e}^{h} \in \mathcal{E}_{\text {disc }_{n}}$. Insertion of (32) into the first equation in (23) results in the final statically condensed system

$\mathbf{K}_{*}^{k} \Delta \mathbf{d}_{n+1}^{k+1}=\mathbf{R}_{*}^{k}$ for the effective residual $\mathbf{R}_{*}=\mathbf{A}_{e=1}^{n_{\text {elem }}} \mathbf{R}_{*}^{e}$ and effective stiffness matrix $\mathbf{K}_{*}=\mathbf{A}_{e=1}^{n_{\text {elem }}} \mathbf{K}_{*}^{e}$ defined by the element contributions

$\mathbf{R}_{*}^{e}=\mathbf{R}^{e}-\mathbf{K}_{d \xi}^{e} \mathbf{K}_{\xi \xi}^{e^{-1}} \mathbf{r}_{e n h}^{e}$ and

$\mathbf{K}_{*}^{e}=\mathbf{K}_{d d}^{e}-\mathbf{K}_{d \xi}^{e} \mathbf{K}_{\xi \xi}^{e^{-1}} \mathbf{K}_{\xi d}^{e}$

only for the elements $\Omega_{e}^{h} \in \mathcal{E}_{d i s c_{n}}$.

The final system of equations (33) involves the nodal displacements only, as desired. We note that, as outlined in the Appendix, the compatibility operator $\mathbf{G}_{(c)}$ in (27) and (29) is designed to capture the strain modes associated to an opening discontinuity correctly, whereas the equilibrium operator $\mathbf{G}_{(e)}$ in (28) and (29) is designed to assure equilibrium along the discontinuity, as discussed in the Appendix. In general we have $\mathbf{G}_{(c)} \neq \mathbf{G}_{(e)}$, a situation that makes the final effective matrix $\mathbf{K}_{*}$ non-symmetric but the final formulation able to capture sharply the strong discontinuities.

Remark 1 During the static condensation and in particular in (32), the inversion of the matrix $\mathbf{K}_{\xi \xi}^{e}$ is required. This matrix, given in (29), is a small matrix consisting of up to $4 \times 4$ entries when all the modes $\xi_{\langle 0 n\rangle}, \xi_{\langle 0 m\rangle}, \xi_{\langle 1 n\rangle}$, and $\xi_{\langle 1 m\rangle}$ are active in the particular finite elements with linear jumps considered in this paper. Its invertibility depends crucially on the definition of the compatibility and equilibrium operators $\mathbf{G}_{(c)}$ and $\mathbf{G}_{(e)}$. For the special case of a fully softened discontinuity, meaning that $\mathbb{C}_{\Gamma}=0$ in (29), and one single node separating for a quadrilateral element a special stabilization treatment is suggested in Linder and Armero (2007) to assure its invertibility.

\section{Representative numerical simulations}

We apply next the finite element formulation outlined above to several problems in dynamic fracture. We consider in particular two benchmark problems, namely, the failure mode transition in ductile materials under impact of Sect. 5.1 and crack branching in brittle materials of Sect. 5.2.

\subsection{Failure mode transition in ductile materials}

Numerous experiments indicate that both propagating shear bands and cracks appear in metals subjected to dynamic loading, with transitions between these failure 


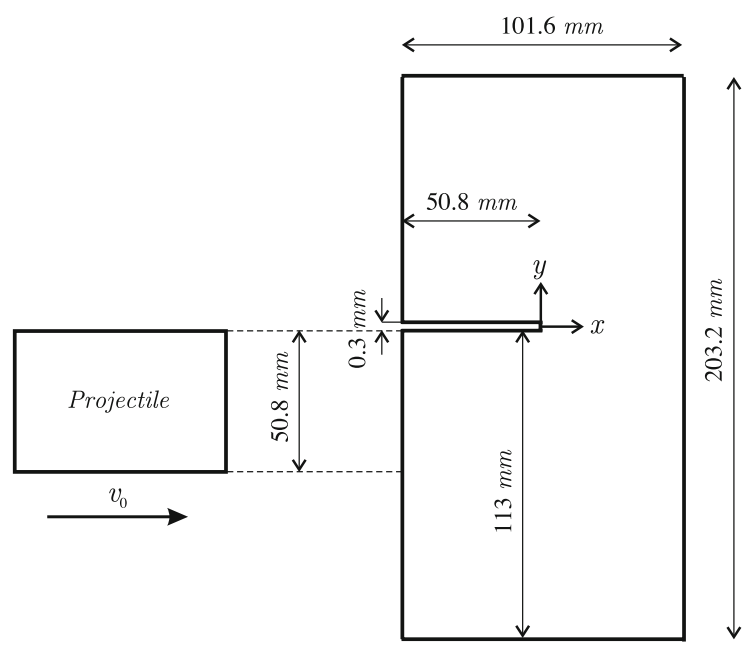

Fig. 5 Failure mode transition in ductile materials: a projectile traveling at the velocity $v_{0}$ impacts the specimen. Subsequently, a shear band starts propagating from the tip of the pre-existing notch almost horizontally. For high impact velocities the shear band propagates through the whole specimen, whereas for low impact velocities the shear band arrests within the specimen resulting in a failure mode transition from ductile to brittle

modes as the loading rate changes. Similar transitions are also observed in materials of a completely different nature, like in polycarbonate (Ravi-Chandar 1995). These failure mode transitions define challenging problems to analyze.

Here we are interested in the particular experimental results presented in Zhou et al. (1996a), where a single notched steel specimen is subjected to the impact of a projectile in the direction of the notch; see Fig. 5 for a representation of the problem to study. This single-notched configuration was also considered in Mason et al. (1994) as a variation of the double-notched plates tested in Kalthoff $(1988,2000)$ and Kalthoff and Winkler (1987). The tests considered a C-300 high strength maraging steel and show a brittle-ductile failure mode transition. A shear band starts propagating from the tip of the notch in the direction parallel to the notch but curving down as it propagates. For low impact velocities, the shear band is observed to arrest and develop a brittle crack at an angle. This is not observed for impact velocities higher than a certain critical value, with the shear band propagating throughout the specimen. We refer to the experimental results presented in Figs. 7 and 8, taken from Zhou et al. (1996a).

These observations present a perfect problem for the validation of numerical methods and, hence, there exists a large number of contributions in the literature considering the numerical simulation of this problem. We can quote Gao and Klein (1999), Falk et al. (2001), Duarte et al. (2001), Klein et al. (2001), Li et al. (2002), Belytschko et al. (2003), Areias and Belytschko (2006), Song et al. (2006), Huespe et al. (2006), Medyanik et al. (2007), Remmers et al. (2008), or Rabczuk and Samaniego (2008), besides the earlier references Needleman and Tvergaard (1994a,b), Belytschko and Tabbara (1996), and Zhou et al. (1996b). The challenge is to accurately capture the experimentally observed failure mode transition from ductile to brittle failure as the impact velocity reduces, as well as the critical value of the impact velocity where this transition occurs.

\subsubsection{Problem setup}

The particular configuration of the problem of interest is illustrated in Fig. 5. The specimen has dimensions $101.6 \mathrm{~mm} \times 203.2 \mathrm{~mm}$ with a pre-existing notch spanning half the width with thickness $0.3 \mathrm{~mm}$. The numerical simulations presented in Zhou et al. (1996b) are performed with finite elements accounting for full thermo-mechanical coupling in the field equations and an elasto-viscoplastic solid at finite strains. In order to simplify the presentation, the temperature and viscosity dependence is not considered in this paper. The specimen is modeled with a von Mises plasticity material model in terms of a Young's modulus of $E=200 \mathrm{GPa}$, a Poisson ratio of $v=0.3$, an uniaxial yield stress of $\sigma_{0}=2 \mathrm{GPa}$, and a density of $\rho=7,830 \mathrm{~kg} / \mathrm{m}^{3}$. Plane strain conditions are assumed.

A shear band starts and propagates from the notch tip when the stresses in the bulk reach the propagation condition

$\|\mathbf{s}\|=\frac{1}{\sqrt{2}}\left|\sigma_{1}-\sigma_{2}\right|$

for the two in-plane principal stresses and the Euclidean norm $\|\mathbf{s}\|$ of the deviatoric stress $\mathbf{s}$. As shown in Armero and Garikipati (1995), this condition corresponds to the loss of ellipticity of the underlying material model. Similarly, this analysis indicates the propagation of the shear band at $45^{\circ}$ with the maximum normal stress direction, choosing the actual direction that corresponds to a given family of slip lines of the two possible such directions; see Armero and Garikipati (1996) for complete details. 


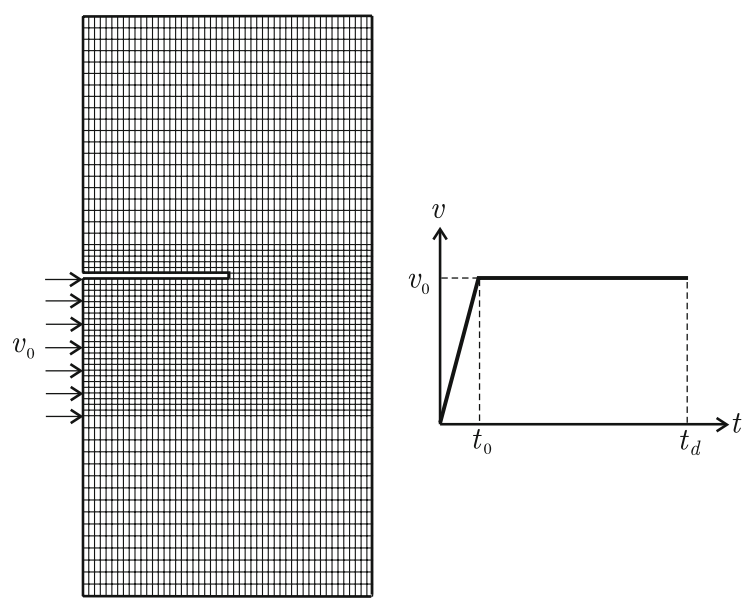

Fig. 6 Failure mode transition in ductile materials: finite element discretization consisting of 3,225 Q1/P0 finite elements. The mesh is refined in the region below the initial crack where the projectile impacts the specimen. The impact is modeled by prescribed velocities along the impact zone based on the diagram shown on the right

After activation, the shear band response is modeled by a localized plastic model with an exponential softening law in the tangential direction in the form

$t_{\Gamma_{m}}=\frac{\llbracket u_{\mu_{m}}^{h} \rrbracket}{\left|\llbracket u_{\mu_{m}}^{h} \rrbracket\right|} \cdot \max \left\{0, \tau_{\max } \cdot \exp \left(-b_{m}\left|\llbracket u_{\mu_{m}}^{h} \rrbracket\right|\right)\right\}$

with a stress threshold $\tau_{\max }=\sigma_{0} / \sqrt{3}$ and an exponent of $b_{m}=1.0 \times 10^{-2} \mathrm{~mm}^{-1}$. Normal opening $\llbracket u_{\mu_{n}}^{h} \rrbracket$ is also allowed, being modeled by the cohesive model

$t_{\Gamma_{n}}=\max \left\{0, \sigma_{\max } \cdot \exp \left(-b_{n} \llbracket u_{\mu_{n}}^{h} \rrbracket\right)\right\}$

with a tensile strength value $\sigma_{\max }=3 \sigma_{0}$ to account for the effect of high triaxiality at the tip of the shear band (Li et al. 2002) and a similar exponential softening law. The softening exponent $b_{n}=3.0 \times 10^{2} \mathrm{~mm}^{-1}$ corresponds to a fracture energy of $G_{f}=20 \mathrm{~kJ} / \mathrm{m}^{2}$, a value of the order typically associated to a C-300 maraging steel (Belytschko et al. 2003). A damage response is assumed in unloading/reloading, with a linear relation to zero traction.

The finite element discretization of the specimen is shown in Fig. 6, where 3,225 Q1/P0 finite elements are used to account for the locking effects in von Mises plasticity models in the bulk. The Q1/P0 mixed interpolation is easily introduced with the corresponding $\overline{\mathbf{B}}$ operator with (average) constant volumetric component; see e.g. Hughes (1987). The finite element mesh is refined in the region where the projectile impacts the specimen and where the shear band location is expected based on the experimental results in Zhou et al. (1996a). The impact of the projectile occurs right below the notch and is modeled by a prescribed velocity on the nodes of the specimen along the impact zone according to the diagram on the right of Fig. 6. After a small rise time of $t_{0}=0.5 \mu \mathrm{s}$ a constant velocity $v_{0}$ is applied until the projectile is supposed to detach from the specimen at $t_{d}=47 \mu \mathrm{s}$. In the numerical simulation the pre-existing notch is modeled with a thickness of $2 \mathrm{~mm}$ instead of the experimental value of $0.3 \mathrm{~mm}$ to prevent the notch faces to exhibit contact during the numerical simulation.

\subsubsection{Numerical results}

In this configuration, the authors in Zhou et al. (1996a) report a critical velocity for the failure mode transition of $v_{\text {crit }}=29 \mathrm{~m} / \mathrm{s}$. For a lower impact velocity $v_{0}<v_{\text {crit }}$ the shear band arrests and kinks at an angle, whereas the shear band propagates throughout the whole specimen for a higher value $v_{0}>v_{\text {crit }}$.

Figures 7 and 8 include the computed paths of the discontinuity for the velocities $v_{0}=25 \mathrm{~m} / \mathrm{s}<v_{\text {crit }}$ and $v_{0}=30 \mathrm{~m} / \mathrm{s}>v_{\text {crit }}$, respectively. For comparison, we have also included a copy of the experimental results reported in Zhou et al. (1996a) for these very same velocities. An overall good agreement between the computed and the experimental results can be observed in both cases. In particular, the numerical simulations capture the observed failure mode transition and for the experimentally measured critical velocity.

For a closer and undistorted comparison, we present in Fig. 9 the final computed path superposed with the experimentally observed path for each impact velocity. The brittle fracture at an angle that occurs for the low velocity of $v_{0}=25 \mathrm{~m} / \mathrm{s}$ can be clearly observed, although the crack appears later than observed in the experiments. The curvature of the shear band can be seen to be well captured in the simulations for both velocities.

Further details of these solutions can be found in Fig. 10, where we have included plots depicting the evolution of the shear band length in time for the two considered velocities, for both the computed solutions and the experiments reported in Zhou et al. (1996a). The overall rates of growth, the slopes in these plots, 
Fig. 7 Failure mode transition in ductile materials: solution for the impact velocity $v_{0}=25 \mathrm{~m} / \mathrm{s}$. a Computed path and $\mathbf{b}$ experimentally observed path as reported in Zhou et al. (1996a) at different times (a)
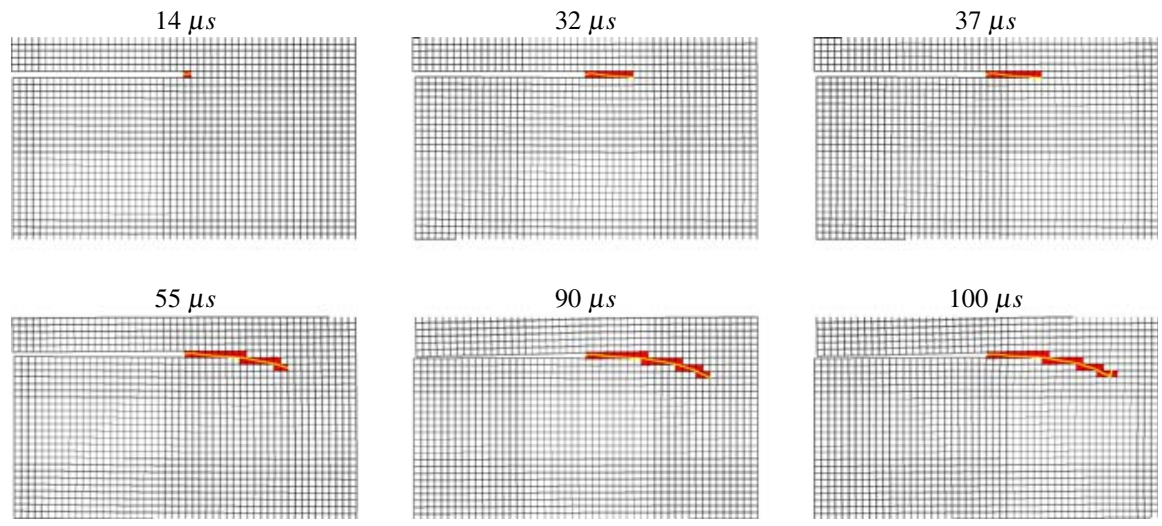

(b)
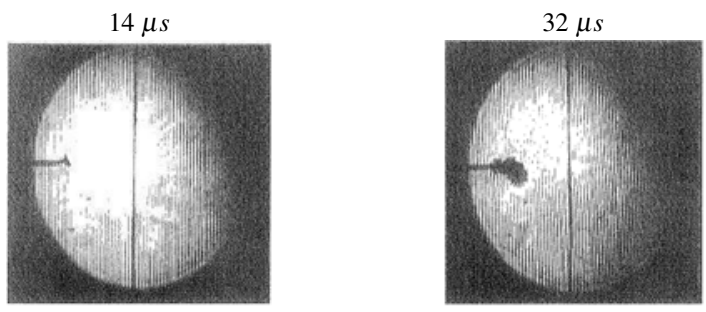

$90 \mu s$

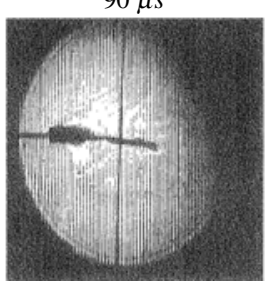

$37 \mu s$

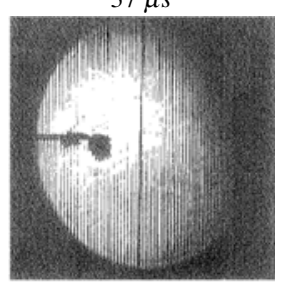

$140 \mu s$

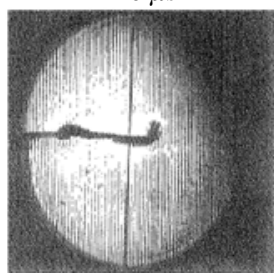

can be seen to be in good agreement, except perhaps for the final stages of the shear band propagation for the higher velocity $v_{0}=30 \mathrm{~m} / \mathrm{s}$ where a faster rate of growth can be observed. The numerical simulations also overestimate the final total length of the shear band when compared with the experimentally observed values. One reason for this may lie in the fact that the experimental results only report the horizontal crack lengths.

We also note that the shear band length is plotted versus the time starting from the initiation of the shear band propagation. The time from initiation is chosen since it differs by approximately $8 \mu$ s when comparing the numerical and experimental results. The initiation starts later in the experiments, a situation that can be attributed to the fact that the measurements do not report the propagation of extremely fine shear bands.
On the other hand the numerical simulation reports the shear band propagation whenever the corresponding slip exceeds machine precision.

Despite these differences, we conclude that an overall good agreement is observed. We note again the capture of the failure mode transition for the exact value of the critical velocity triggering it, with both the brittle and ductile responses of the material resolved by the new finite elements with embedded discontinuities developed in this work.

\subsection{Crack branching in brittle materials}

We consider next the problem of crack branching in brittle materials. This challenging problem has become a benchmark for the computational modeling 
Fig. 8 Failure mode transition in ductile materials: solution for the impact velocity $v_{0}=30 \mathrm{~m} / \mathrm{s}$. a Computed path and $\mathbf{b}$ experimentally observed path as reported in Zhou et al. (1996a) at different times (a)

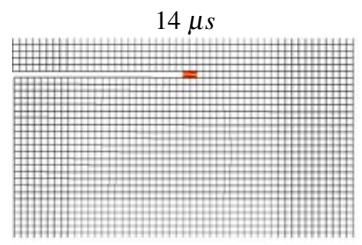

$47 \mu s$

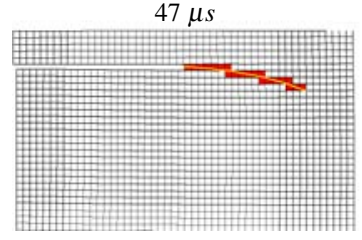

(b)

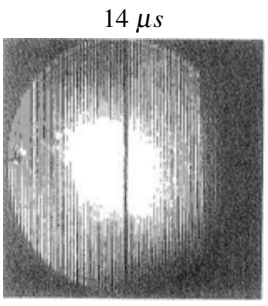

$47 \mu s$

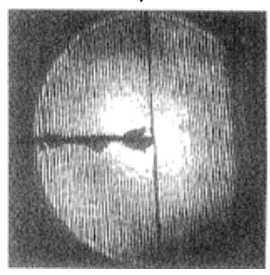

$39 \mu s$

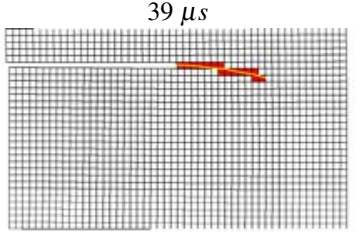

$51 \mu s$

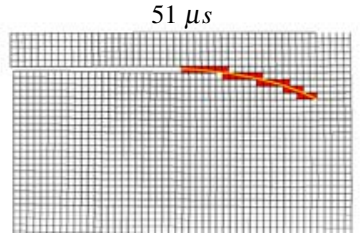

$39 \mu s$

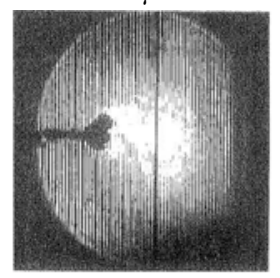

$51 \mu s$

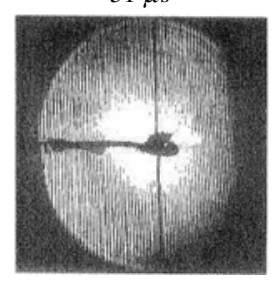

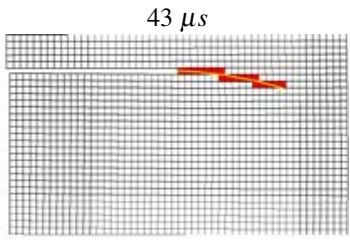

$60 \mu s$

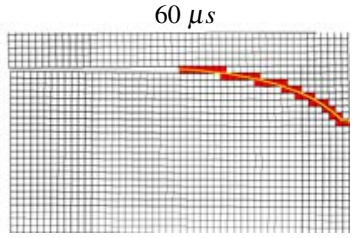

$43 \mu s$

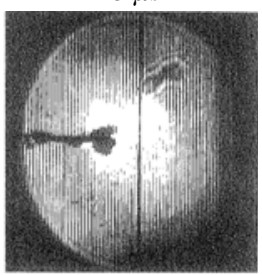

$64 \mu s$

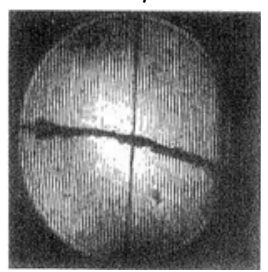

of dynamic fracture. We refer among others to Dally (1979), Ravi-Chandar and Knauss (1984a,b), Knauss and Ravi-Chandar (1985), Ramulu and Kobayashi (1985), Fineberg et al. (1991), Satoh (1996), Sharon and Fineberg (1996, 1999), Fineberg and Marder (1999), and references therein, for a discussion of some experimental observations. Numerical simulations of the problem have been reported in Falk et al. (2001), Klein et al. (2001), Belytschko et al. (2003), Zhou and Molinari (2004), Zhou et al. (2005), Huespe et al. (2006), Song et al. (2006), Duarte et al. (2007), Karedla and Reddy (2007), Remmers et al. (2008), Zhang et al. (2007), Zi et al. (2007), to mention just a few references employing a variety of different numerical approaches. Here we present complete comparisons with the numerical results reported in $\mathrm{Xu}$ and Needleman (1994) and
Falk et al. (2001), given the ability of complete details of the problems setup.

\subsubsection{Problem setup}

We consider a center cracked rectangular block in plane strain with dimensions of $6 \mathrm{~mm} \times 3 \mathrm{~mm}$ and an initial central crack of length $0.6 \mathrm{~mm}$ as illustrated in Fig. 11. The loading is applied at the top and bottom faces at $y= \pm 1.5 \mathrm{~mm}$ based on the diagram given on the right of that figure. The applied velocity $v$ increases linearly from 0 at time $t=0$, at which the specimen is stress free and at rest, to $v_{0}$ at time $t=0.1 \mu \mathrm{s}$ and remains constant thereafter. Different values for this imposed velocity $v_{0}$ are considered during the numerical simulations below. 
Fig. 9 Failure mode transition in ductile materials: Comparison of the shear band location for different impact velocities observed in experiments (Zhou et al. 1996a) (solidline) with the results obtained in this work (dashedline). The result for $v_{0}=25 \mathrm{~m} / \mathrm{s}$ is shown on the left, whereas the result for $v_{0}=30 \mathrm{~m} / \mathrm{s}$ is shown on the right

Fig. 10 Failure mode transition in ductile materials: shear band length versus time for different impact velocities observed in experiments (Zhou et al. 1996a) and this work. The result for $v_{0}=25 \mathrm{~m} / \mathrm{s}$ is shown on the left, whereas the result for $v_{0}=30 \mathrm{~m} / \mathrm{s}$ is shown on the right
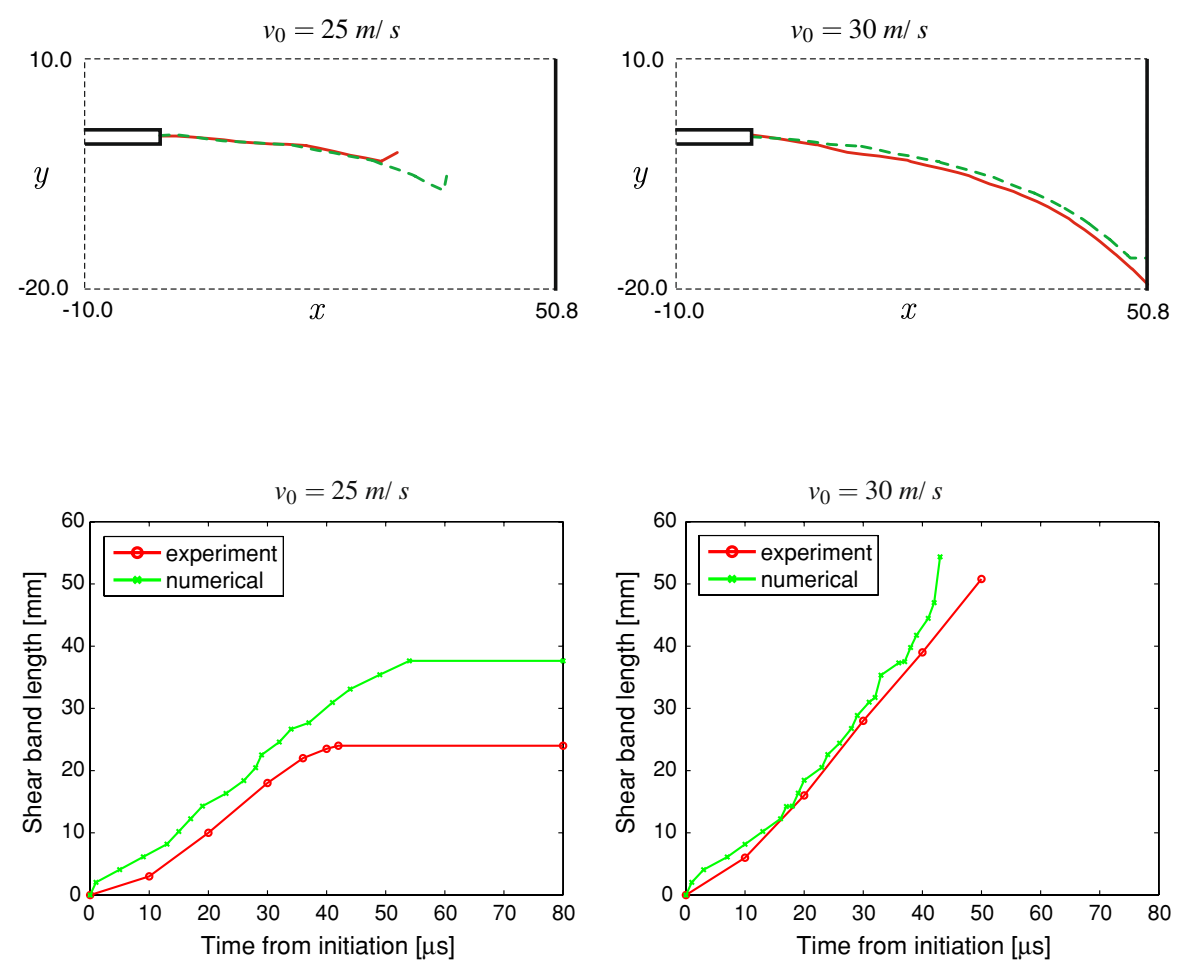

The brittle material corresponds to polymethylmethacrylate (PMMA), which we model with a linear elastic material until reaching a maximum normal stress $\sigma_{\max }$. A discontinuity is then activated in the direction orthogonal to that maximum principal stress. We assume the linear cohesive laws

$t_{\Gamma_{n}}=\max \left\{0, \sigma_{\max }+S_{n} \llbracket u_{\mu_{n}}^{h} \rrbracket\right\}$

and

$t_{\Gamma_{m}}=\frac{\llbracket u_{\mu_{m}}^{h} \rrbracket}{\left|\llbracket u_{\mu_{m}}^{h} \rrbracket\right|} \cdot \max \left\{0, \tau_{\max }+S_{m}\left|\llbracket u_{\mu_{m}}^{h} \rrbracket\right|\right\}$

between the opening displacements $\llbracket u_{\mu_{n}}^{h} \rrbracket$ and $\llbracket u_{\mu_{m}}^{h} \rrbracket$ in normal and tangential directions to the corresponding traction components in those directions $t_{\Gamma_{n}}$ and $t_{\Gamma_{m}}$, respectively, along the discontinuities during loading. We consider again a linear damage response in unloading/reloading.

The assumed material parameters for the elastic response of the bulk are $E=3.24 \mathrm{GPa}$ for the Young's modulus, $v=0.35$ for the Poisson ratio, and $\rho=1,190 \mathrm{~kg} / \mathrm{m}^{3}$ for the density. These values result in the wave speeds

$$
\begin{aligned}
& c_{d}=\sqrt{\frac{E(1-v)}{\rho(1+v)(1-2 v)}}=2,090 \mathrm{~m} / \mathrm{s} \\
& c_{s}=\sqrt{\frac{E}{2 \rho(1+v)}}=1,004 \mathrm{~m} / \mathrm{s} \\
& c_{R}=c_{s} \frac{0.862+1.14 v}{1+v}=938 \mathrm{~m} / \mathrm{s}
\end{aligned}
$$

for the dilational, shear, and Rayleigh, respectively (Freund 1998). For the localized cohesive laws, we consider the values $\sigma_{\max }=\tau_{\max }=E / 25=129.6 \mathrm{MPa}$ and a linear softening modulus of $S_{n}=S_{m}=$ $-24 \mathrm{GPa} / \mathrm{mm}$. These values correspond to a fracture energy of $G_{f}=0.35 \mathrm{~kJ} / \mathrm{m}^{2}$, as it is characteristic of PMMA (Sharon and Fineberg 1999).

We consider a finite element discretization consisting of $40 \times 41$ standard displacement-based bilinear quadrilaterals for the half specimen $x \geq 0$ due to the symmetry in the geometry and the loading. To take into account the pre-existing central crack the elements with centroids $\left(x_{c}, y_{c}\right)$ at $y_{c}=0$ and $x_{c}<0.3 \mathrm{~mm}$ are approximated with the traction separation laws along 
Fig. 11 Crack branching in brittle materials: geometry of the center cracked block (left) together with the loading history in terms of the applied velocity $v$ at the top and the bottom faces of the block (right). The crack is expected to propagate horizontally towards the left and right edges of the block. After a certain extension crack branching is expected

Fig. 12 Crack branching in brittle materials: crack length (left) and normalized crack tip velocities ( $r i g h t$ ) versus time for applied velocities of $v_{0}=3,5,7,10,15 \mathrm{~m} / \mathrm{s}$ without branching allowed
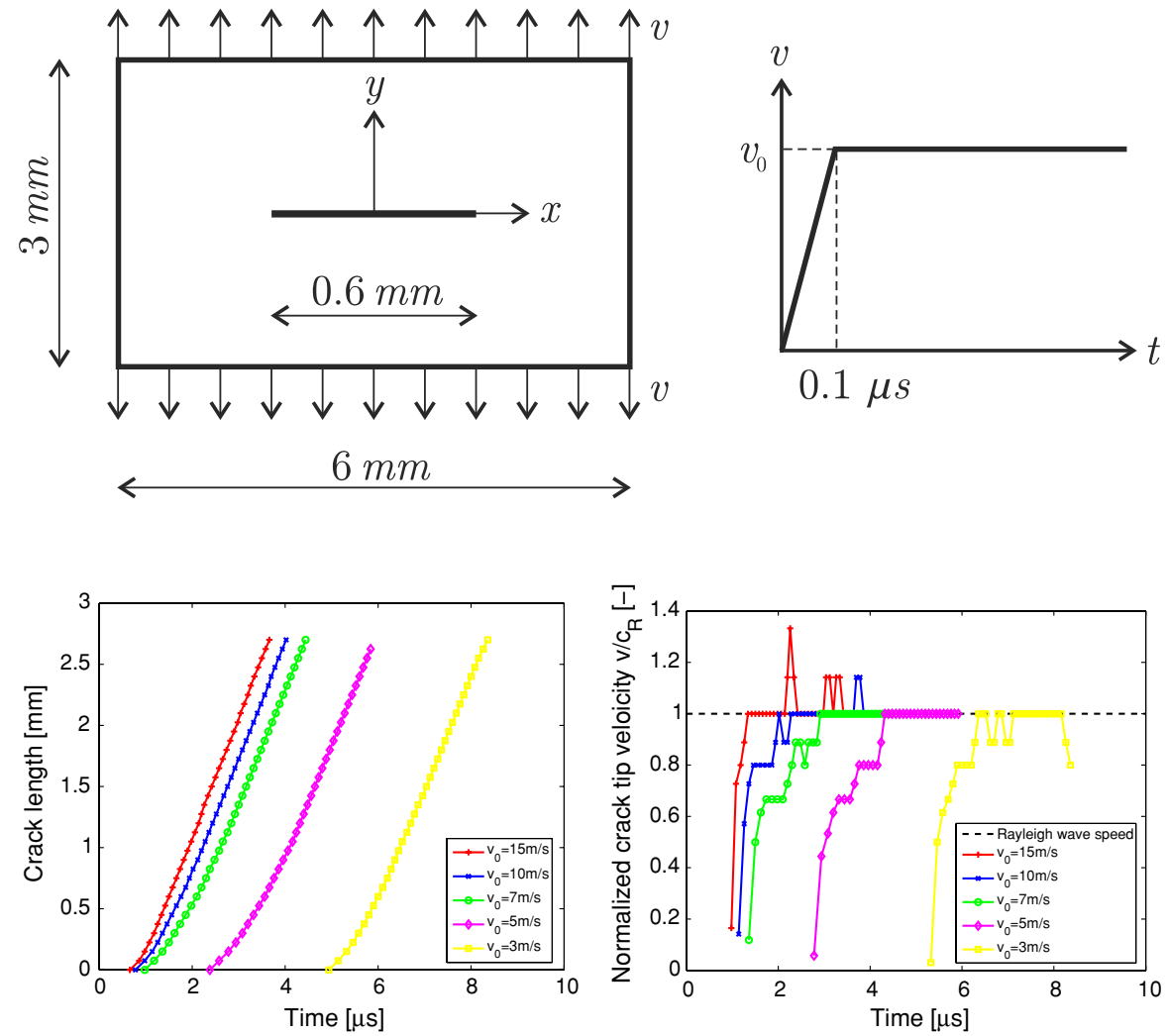

the strong discontinuity (38) and (39) with vanishing tractions $t_{\Gamma_{n}}$ and $t_{\Gamma_{m}}$ in the normal and tangential directions, that is, $t_{\Gamma_{n}}=t_{\Gamma_{m}}=0$ in the elements capturing the pre-existing strong discontinuity along $0 \leq x \leq$ $0.3 \mathrm{~mm}$.

\subsubsection{Crack tip velocity as branching criterion}

When performing the numerical simulations for different velocities $v_{0}$ with the proposed finite elements and the discretization outlined above, the initial crack tip at $x=0.3 \mathrm{~mm}$ propagates horizontally until it fully crosses the whole specimen and reaches the right edge at $x=3 \mathrm{~mm}$. When plotting the crack tip velocities for applied velocities of $v_{0}=3,5,7,10,15 \mathrm{~m} / \mathrm{s}$ in Fig. 12 one can observe that some measured crack tip velocities surpass the Rayleigh wave speed, but we obtain this theoretical limit for this problem (Freund 1998) in most cases. No branching is allowed in these runs. However, experiments (Ravi-Chandar and Knauss 1984a,b; Knauss and Ravi-Chandar 1985; Sharon and Fineberg 1996, 1999) and analytical investigations (Yoffe 1951; Eshelby 1970; Gao 1993, 1996) predict that after exceeding a certain critical ratio $v_{\text {crit }} / c_{R}<1$, the crack tends to branch rather than continuing its motion in a straight path, reducing its speed. Interestingly, the analytical studies tend to overestimate this critical ratio when compared with the experimental observations (Ravi-Chandar and Knauss 1984b; Knauss and Ravi-Chandar 1985; Gao 1993, 1996; Fineberg and Marder 1999).

In order to capture the branching, the crack tip velocity is employed as a branching criterion in this work. That means that branching is initiated when the crack tip velocity reaches a critical velocity $v_{\text {crit }}$. The actual triggering of the branching is obtained by stopping the main crack and initiating cracks at the center of the edges of the adjacent top and bottom elements instead with respect to the previous main branch.

In the actual numerical implementation, based on a front propagating through the connectivity graph of the mesh (the front corresponding to a finite element candidate for the advancement of a discontinuity), branching reduces to the start of two new fronts. 
Fig. 13 Crack branching in brittle materials: illustration of the crack path for an applied velocity of $v_{0}=3 \mathrm{~m} / \mathrm{s}$. Results obtained in Falk et al. (2001) are shown on the left whereas results obtained in this work at time $t=8.7 \mu \mathrm{s}$ are shown on the right

Fig. 14 Crack branching in brittle materials: crack length (left) and normalized crack tip velocities (right) versus time for an applied velocity of $v_{0}=3 \mathrm{~m} / \mathrm{s}$. A comparison with the results in Falk et al. (2001) is illustrated
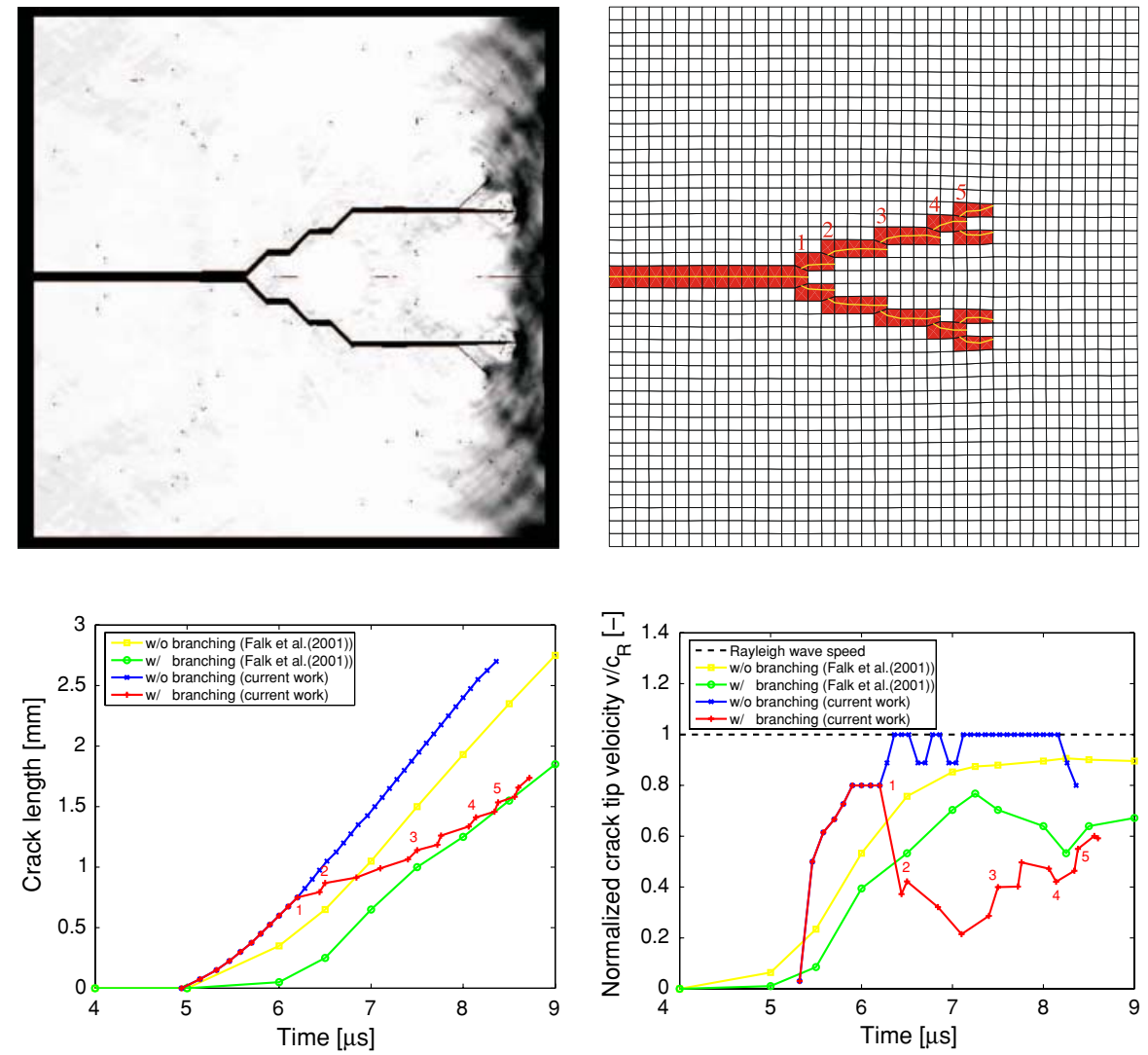

It is up to the evaluation of the state of stress in those front elements to actually propagate the discontinuity in a particular direction, thus propagating the front. In fact, the propagation of fronts is carried out for as many elements where the propagation condition is satisfied (i.e. maximum normal stress reaching the material's tensile strength in this particular problem). If the propagating front encounters an element with an existing active discontinuity, the propagating front stops, as one can envisage occurring in the actual setting of a crack encountering another open crack.

The crack tip velocity is easily evaluated from the progress of the discontinuity path, and the contiguous elements are simply given by the mesh connectivity. We use critical ratios of $v_{\text {crit }} / c_{R}=0.7-0.8$ in the numerical simulations reported below in our goal to compare the current formulation with the published numerical results of Xu and Needleman (1994) and Falk et al. (2001). These values are relatively high for the experimental observations of branching in PMMA (Sharon and Fineberg 1996, 1999; Fineberg and Marder 1999) for the considered problem. We refer to Linder and Armero (2009) for a study of the influence of the critical ratio $v_{\text {crit }} / c_{R}$ in the numerical simulations.

\subsubsection{Numerical results}

We consider impact velocities of $v_{0}=3,5,15 \mathrm{~m} / \mathrm{s}$. Figures 13, 14, 15, 16, 17 and 18 show the crack paths together with the corresponding computed evolutions in time of the crack length and the crack tip velocity for these different impact velocities.

More specifically, Figs. 13 and 14 show the results obtained for the impact velocity $v_{0}=3 \mathrm{~m} / \mathrm{s}$. Figure 13 compares the crack path obtained in the current work with the path obtained in Falk et al. (2001). We can observe a good agreement in the location of the crack branching. Also, a similar behavior is observed after the branching occurs. The crack tip velocity reaches $80 \%$ of the Rayleigh wave speed at $t=6.2 \mu \mathrm{s}$ at a crack length of about $0.825 \mathrm{~mm}$ (without counting 
Fig. 15 Crack branching in brittle materials: illustration of the crack path for an applied velocity of $v_{0}=5 \mathrm{~m} / \mathrm{s}$. Results obtained in $\mathrm{Xu}$ and Needleman (1994) are shown on the left whereas results obtained in this work at time $t=6.5 \mu \mathrm{s}$ are shown on the right
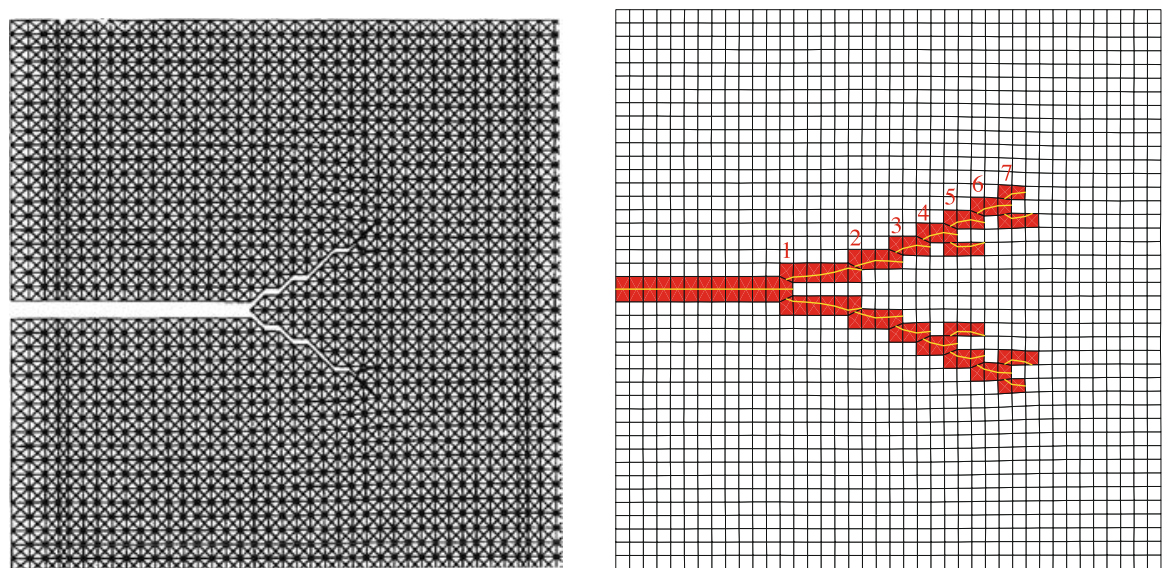

Fig. 16 Crack branching in brittle materials: crack length (left) and normalized crack tip velocities ( $r i g h t$ ) versus time from initial crack initiation, which occurs at a total time of $t=2.77 \mu \mathrm{s}$ in the current work, for an applied velocity of $v_{0}=5 \mathrm{~m} / \mathrm{s}$. A comparison with the results in $\mathrm{Xu}$ and Needleman (1994) is illustrated for the crack tip velocity
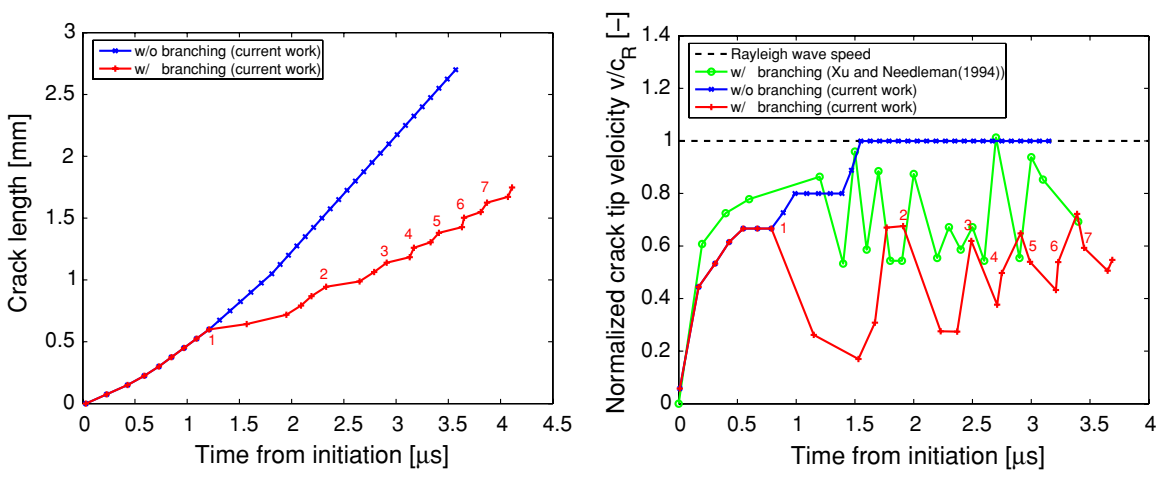

the pre-existing crack length of $0.3 \mathrm{~mm}$ ) in the current work. The numbering 1-5 in Figs. 13 and 14 allows for a comparison of the different stages monitored during the crack branching process. Looking at the branching pattern one can identify two main crack branches originating from the single branch at point 1 . The critical velocity $v_{\text {crit }}$ is again reached at points $2-4$, with the program activating two branches but one of the branches can be seen not to propagate. Only at the end of the simulation, at point 5 , crack branching is again detected and the two activated branches propagate.

Figure 14 compares the evolutions in time of the crack length and crack tip velocity obtained in this work, with and without branching, with the results reported in Falk et al. (2001). When branching is suppressed the crack tip velocity increases up to the Rayleigh wave speed and remains almost constant with the crack horizontally propagating towards the right end of the specimen. Looking at the crack tip velocities with branching, one can observe a faster increase in the results of the current work when compared to the results in Falk et al. (2001). On the other hand the overall behavior is very similar, that is, without branching the Rayleigh wave speed is approached with the crack tip velocity remaining almost constant in that case, whereas with branching the crack tip velocity slows down significantly after branching.

Similar results are obtained for the imposed velocity of $v_{0}=5 \mathrm{~m} / \mathrm{s}$, as depicted in Figs. 15 and 16. In Fig. 15 we compare the computed crack path in this work with the crack path reported in Xu and Needleman (1994).

This reference uses cohesive elements across the pre-existing element edges of the mesh to model the opening of the crack.

We can observe again a good agreement of the location of the initial crack branching. The crack tip velocity reaches the critical velocity at total time $t=3.56 \mu \mathrm{s}$ (i.e. $t=0.79 \mu$ s after the initiation of the first crack branch) at a crack length of $0.675 \mathrm{~mm}$ (again without counting the pre-existing crack length of $0.3 \mathrm{~mm}$ ). We also observe a similar response after branching even though the branching angle we obtain in this work 
Fig. 17 Crack branching in brittle materials: illustration of the crack path for an applied velocity of $v_{0}=15 \mathrm{~m} / \mathrm{s}$. Results obtained in $\mathrm{Xu}$ and Needleman (1994) are shown on the left whereas results obtained in this work at time $t=3.2 \mu \mathrm{s}$ are shown on the right
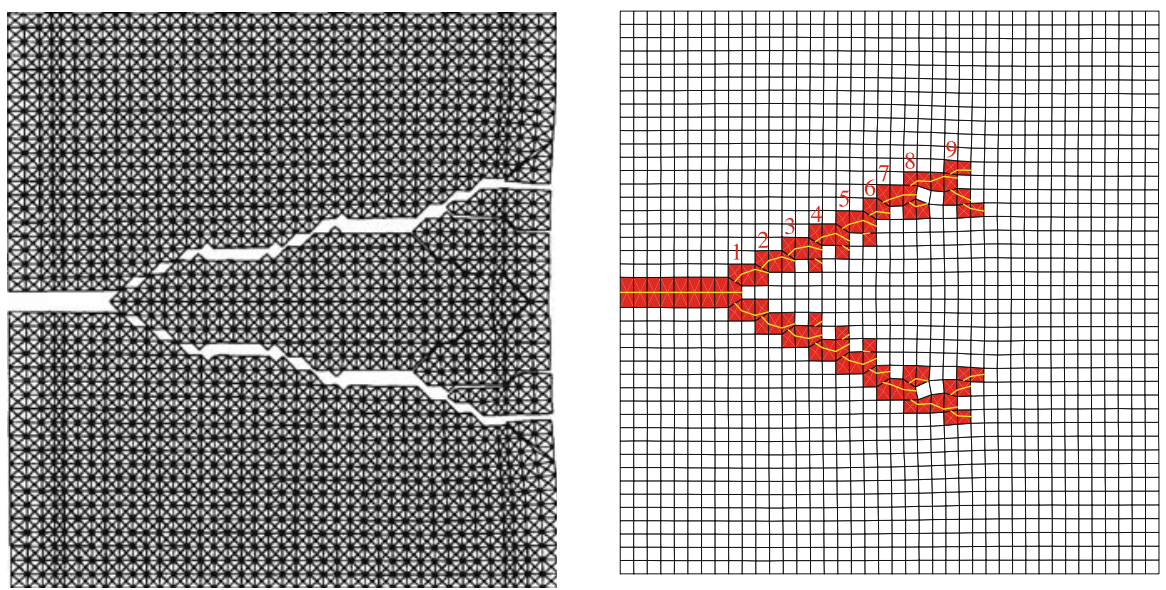

Fig. 18 Crack branching in brittle materials: crack length $($ left $)$ and normalized crack tip velocities (right) versus time for an applied velocity of $v_{0}=15 \mathrm{~m} / \mathrm{s}$

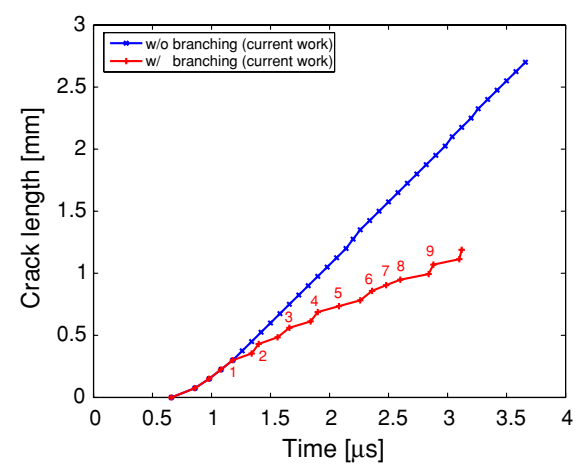

is smaller. Figure 16 compares crack tip velocities of the current work with and without branching with the results provided in Xu and Needleman (1994). A good agreement is obtained for the evolution in time of the crack tip velocity. We consider again the time starting from the initiation of the crack.

We follow the same steps in Figs. 17 and 18 for the imposed velocity of $v_{0}=15 \mathrm{~m} / \mathrm{s}$, including the crack path obtained in Xu and Needleman (1994). In this case, the crack tip velocity reaches the critical velocity at the total time $t=1.18 \mu \mathrm{s}$ and at a crack length of $0.375 \mathrm{~mm}$ (again without counting the pre-existing crack length of $0.3 \mathrm{~mm}$ ). The location of the crack branching agrees very well with the results reported in this reference, whereas the branching angle is steeper in the current work. Figure 18 shows the crack lengths and crack tip velocities without and with branching we have obtained with the finite elements developed in this work; no data of crack tip velocity for $v_{0}=15 \mathrm{~m} / \mathrm{s}$ is provided in $\mathrm{Xu}$ and Needleman (1994).
Note that the results of the crack lengths and crack tip velocities in Figs. 14, 16, and 18 are always reported for the very top branches of the branching crack. Symmetry applies about the horizontal middle line, as confirmed by the computed crack paths. The overall branching angles obtained for the different velocities of $v_{0}=3,5,15 \mathrm{~m} / \mathrm{s}$ are $\sim 21^{\circ}, 22^{\circ}, 28^{\circ}$ off the horizontal axis, respectively. We conclude that bigger branching angles appear for higher applied velocities $v_{0}$, in good agreement with experimental observations.

To illustrate the influence of the mesh size on the solution, we consider again the first case of this problem, namely, an imposed opening velocity of $v_{0}=$ $3 \mathrm{~m} / \mathrm{s}$, but with a finer mesh consisting of $50 \times 51$ elements. Figures 19 and 20 depict the solutions obtained for this case. In particular, this last figure shows the computed pattern of the branching, a pattern to be compared with the solution on the right side of Fig. 13. Figure 19 carries this comparison in more detail by showing the evolution in time of the crack length and 
Fig. 19 Crack branching in brittle materials: crack length (left) and normalized crack tip velocities ( $r i g h t)$ versus time for an applied velocity of $v_{0}=3 \mathrm{~m} / \mathrm{s}$. Comparison for two meshes with different level of discretization. The meshes and branching patterns are shown in Figs. 13 (right) and 20 for the coarse and fine meshes, respectively
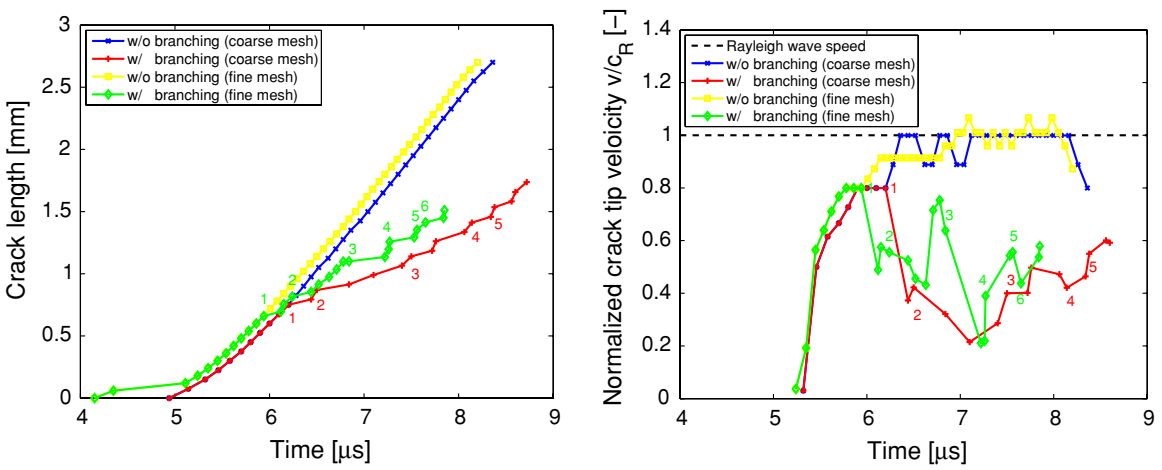

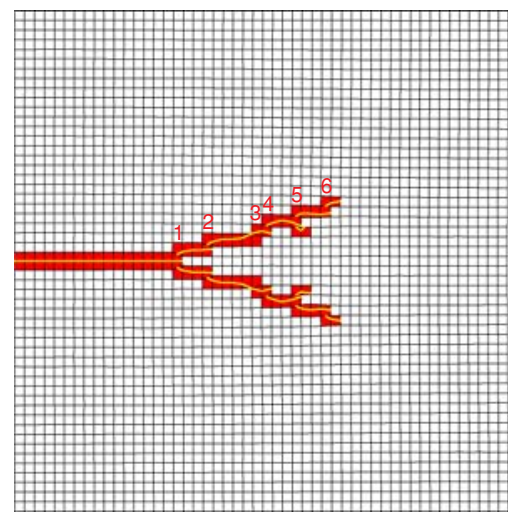

Fig. 20 Crack branching in brittle materials: illustration of the crack path for an applied velocity of $v_{0}=3 \mathrm{~m} / \mathrm{s}$. Results obtained for a fine mesh to check the sensitivity of the results on the mesh size. This solution is to be compared with the one shown on the right of Fig. 13 for the original coarser mesh

relative crack tip velocity for both the new mesh and the original coarser mesh, as reported in Fig. 14. We have also included the solutions obtained with no branching allowed, recovering again the theoretical limit of the Rayleigh speed for this problem.

Overall we can observe a good agreement between the solutions obtained with the two different meshes (compare, for example, the branching angles), with some differences in the details, as one would expect in the simulations of these difficult problems. We can observe, for instance, that the different branches appear slightly earlier in time for the finer mesh. We note that the considered finite element formulation is fully objective with respect to mesh size (that is, it does not exhibit the pathological mesh dependence typical of continuum elements due to the incorrect resolution of the localized dissipative mechanism associated with fracture) as illustrated in detail in Linder and Armero (2007).

\section{Concluding remarks}

We have presented in this paper the extension of finite elements with embedded strong discontinuities to the dynamic range for the study and simulation of dynamic fracture. The numerical results presented here show that this formulation is able to capture well different aspects characteristic of these problems. In particular, we have shown that brittle/ductile failure mode transitions and crack branching can be captured well with these elements.

The actual finite elements considered in the simulations are new quadrilateral finite elements developed in this framework incorporating a linear separating discontinuity. The extension of these finite elements, originally developed and tested in the quasi-static case, to the general dynamic range has been accomplished very easily through the incorporation of the inertial effects in the mechanical initial boundary value problem of the global problem, not affecting the local modeling and approximation of the strong discontinuity. This observation outlines again the appropriateness of the considered multi-scale framework for the treatment of strong discontinuities in solids by which these solutions are treated in a global/local framework. Due to this, the other big advantages of this framework are fully maintained in the dynamic range considered here, namely, the high computational efficiency of the final formulation (with a local elimination of any additional degrees of freedom capturing the discontinuities) and the simplicity of its incorporation in an existing and general finite element code (involving local considerations at the element level).

Our current and future work in this area is focused on further extensions of the finite element formulations considered here. In particular, we are exploring the formulation of finite elements that treat crack branching 
in their interiors. Crucial to the success of these formulations is the approach followed in the design of the enhanced strain fields capturing the effect of the discontinuity based, as illustrated in this paper, on the incorporation of the correct discrete strains in the element rather than on the incorporation of complex local interpolation of a discontinuous displacement field. Preliminary results of these considerations can already be found in Linder and Armero (2009). Additional extensions under further study include the consideration of finite deformation effects and other effects significant to the problems presented here.

Acknowledgments Financial support for this research was provided by the AFOSR under Grant no. FA9550-08-1-0410 with UC Berkeley. This support is gratefully acknowledged.

Open Access This article is distributed under the terms of the Creative Commons Attribution Noncommercial License which permits any noncommercial use, distribution, and reproduction in any medium, provided the original author(s) and source are credited.

\section{Appendix}

1 Closed form expression of the equilibrium operator $\mathbf{G}_{(e)}$

As discussed in Sect. 3.2, the equilibrium operator $\mathbf{G}_{(e)}$ can be understood as a projection operator taking the stresses in the bulk of the element $\Omega_{e}^{h}$ (in fact, the quadrature points in the bulk) to the corresponding tractions on the discontinuity segment $\Gamma_{e}^{h}$. Comparing the local equilibrium Eq. (13) with its discrete counterpart (18), we must have

$$
\int_{\Omega_{e}^{h}} \mathbf{G}_{(e)}^{\langle k\rangle^{T}} \sigma \mathrm{d} \Omega=-\int_{\Gamma_{e}^{h}} s^{k} \sigma \mathbf{n} d \Gamma+l_{\Gamma_{e}^{h}} o\left(h_{e}^{p+1}\right)
$$

for a rate of accuracy $p \geq 0$, with $k=0,1, \ldots, q \leq p$ corresponding to the polynomial degree assumed for the interpolation of the displacement jump along $\Gamma_{e}^{h}$ [e.g. $q=1$ for the linear interpolation (16)]. Here we have introduced the notation $h_{e}=A_{\Omega_{e}^{h}} / l_{\Gamma_{e}^{h}}$ following the original definitions (14) for element $\Omega_{e}^{h}$.

For the case of interest here $q=1$ (linears), we write

$$
\mathbf{G}_{(e)}=\left[\begin{array}{llll}
\mathbf{G}_{(e)}^{\langle 0 n\rangle} & \mathbf{G}_{(e)}^{\langle 0 m\rangle} & \mathbf{G}_{(e)}^{\langle 1 n\rangle} & \mathbf{G}_{(e)}^{\langle 1 m\rangle}
\end{array}\right]
$$

with the individual contributions given by

$$
\begin{aligned}
\mathbf{G}_{(e)}^{\langle k n\rangle} & =-\frac{1}{h_{e}} g_{e}^{\langle k\rangle}(x, y)(\mathbf{n} \otimes \mathbf{n}) \text { and } \\
\mathbf{G}_{(e)}^{\langle k m\rangle} & =-\frac{1}{h_{e}} g_{e}^{\langle k\rangle}(x, y)(\mathbf{n} \otimes \mathbf{m})^{s}
\end{aligned}
$$

for $k=0,1$, with the functions $g_{e}^{\langle k\rangle}(x, y)$ defined in a local Cartesian system $\{x, y\}$ of the element. As argued originally in Armero and Ehrlich (2006b) in the context of plate elements, these functions can be constructed with polynomials. In this way, we write

$g_{e}^{\langle k\rangle}(x, y)=a_{(0,0)}^{\langle k\rangle}+a_{(1,0)}^{\langle k\rangle} x+a_{(0,1)}^{\langle k\rangle} y$

with

$$
\begin{aligned}
& {\left[\begin{array}{c}
a_{(0,0)}^{\langle k\rangle} \\
a_{(1,0)}^{k k\rangle} \\
a_{(0,1)}^{\langle k\rangle}
\end{array}\right]=\left(\frac{1}{A_{\Omega_{e}^{h}}} \int_{\Omega_{e}^{h}}\left[\begin{array}{ccc}
1 & x & y \\
x & x^{2} & x y \\
y & x y & y^{2}
\end{array}\right] \mathrm{d} \Omega\right)^{-1}} \\
& \left(\frac{1}{l_{\Gamma_{e}^{h}}} \int_{\Gamma_{e}^{h}}\left[\begin{array}{c}
s^{k} \\
s^{k} x \\
s^{k} y
\end{array}\right] \mathrm{d} \Gamma\right)
\end{aligned}
$$

again for the linear case $q=1$. It is important to note that all these terms are completely defined once the discontinuity element is propagated through element $\Omega_{e}^{h}$, so these coefficients only need to be computed once. Note also that the integrals in (46) are evaluated using the quadrature rules (19).

2 Closed form expression of the enhanced strain operator $\mathbf{G}_{(c)}$

The enhanced operator $\mathbf{G}_{(c)}$ defining the small scale strains (20) must capture the effects of the strong discontinuity in the bulk of the element. In particular, and as argued in Sect. 3.2, this operator must be such that the linear separation mode depicted in Fig. 4 is captured for any opening $\xi$. This assures, in particular, that no additional strains (and so stresses) appear in this opening mode, that is, avoiding stress-locking.

Denoting the two parts of the element separated by a discontinuity $\Gamma_{e}^{h}$ by $\Omega_{e}^{h^{+}}$and $\Omega_{e}^{h^{-}}$the separation mode in Fig 4 is characterized by the nodal displacements

$\mathbf{d}_{\mathrm{A}, \text { mode }}(\boldsymbol{\xi})= \begin{cases}\xi_{\langle 0 n)} \boldsymbol{n}+\xi_{\langle 0 m\rangle} \boldsymbol{m} & \text { for } A \in \Omega_{e}^{h^{+}} \\ +\boldsymbol{L}\left(\boldsymbol{\xi}_{\langle 1\rangle}\right) \overline{\boldsymbol{x}}_{A} & \\ 0 & \text { otherwise }\end{cases}$

for $\overline{\boldsymbol{x}}_{A}=\boldsymbol{x}_{A}-\boldsymbol{x}_{\Gamma}$, and the interpolation matrix

$\boldsymbol{L}\left(\boldsymbol{\xi}_{\langle 1\rangle}\right)=\xi_{\langle 1 n\rangle}(\boldsymbol{n} \otimes \boldsymbol{m})^{a}+\xi_{\langle 1 m\rangle}(\boldsymbol{m} \otimes \boldsymbol{m})$ 
corresponding to the constant and linear parts of the jumps as considered in (16). The interpolation matrix $\boldsymbol{L}\left(\boldsymbol{\xi}_{\langle 1\rangle}\right)$ corresponds to an infinitesimal rotation of the separating part (note that $(\cdot)^{a}$ denotes the skewsymmetric part) as well as a stretching in tangential direction to $\Gamma_{e}^{h}$. The nodal displacements (48) must come with the small-scale strain

$\boldsymbol{\varepsilon}_{\mu_{\text {mode }}}^{h}=\xi_{\langle 1 m\rangle}(\boldsymbol{m} \otimes \boldsymbol{m}) \mathcal{H}_{\Gamma_{e}^{h}}\left(\boldsymbol{x}^{h}\right)$

for the Heaviside jump function $\mathcal{H}_{\Gamma_{e}^{h}}\left(\boldsymbol{x}^{h}\right)$ (= 1 for $x^{h} \in \Omega_{e}^{h^{+}}$, vanishing otherwise), corresponding to the stretching of $\Omega_{e}^{h^{+}}$in the tangential direction $\boldsymbol{m}$.

Inserting relations (48-50) in (20) and imposing that the final relation is satisfied by any enhanced parameters $\boldsymbol{\xi}$ results in the sought enhanced operator $\mathbf{G}_{(c)}$, which can be written as

$\mathbf{G}_{(c)}=\left[\begin{array}{llll}\mathbf{G}_{(c)}^{\langle 0 n\rangle} & \mathbf{G}_{(c)}^{\langle 0 m\rangle} & \mathbf{G}_{(c)}^{\langle 1 n\rangle} & \mathbf{G}_{(c)}^{\langle 1 m\rangle}\end{array}\right]$

with

$$
\begin{aligned}
\mathbf{G}_{(c)}^{\langle 0 n\rangle} & =-\sum_{A \in \Omega_{e}^{h^{+}}} \overline{\mathbf{B}}^{A} \boldsymbol{n} \quad \text { and } \\
\mathbf{G}_{(c)}^{\langle 0 m\rangle} & =-\sum_{A \in \Omega_{e}^{h^{+}}} \overline{\mathbf{B}}^{A} \boldsymbol{m}
\end{aligned}
$$

for the constant jump contributions, and with

$$
\mathbf{G}_{(c)}^{\langle 1 n\rangle}=-\sum_{A \in \Omega_{e}^{h^{+}}} \overline{\mathbf{B}}^{A}(\boldsymbol{n} \otimes \boldsymbol{m})^{a} \overline{\boldsymbol{x}}_{A}
$$

and

$$
\mathbf{G}_{(c)}^{\langle 1 m\rangle}=(\boldsymbol{m} \otimes \boldsymbol{m}) \mathcal{H}_{\Gamma_{e}^{h}}-\sum_{A \in \Omega_{e}^{h^{+}}} \overline{\mathbf{B}}^{A}(\boldsymbol{m} \otimes \boldsymbol{m}) \overline{\boldsymbol{x}}_{A}
$$

for the linear jump contributions. We note the presence of the assumed strain operator $\overline{\mathbf{B}}$, for whatever the underlying finite element is. We refer to Linder and Armero (2007) for complete details on these enhanced operators for strong discontinuities, and to Armero and Linder (2008) for the extension of these considerations to the finite deformation case.

Remark 2 As noted in the end of Sect. 3.2 above, the approach followed here does not require the consideration of a discontinuous small-scale displacement $\boldsymbol{u}_{\mu}^{h}\left(\boldsymbol{x}^{h}\right)$. However, such a local displacement distribution can be obtained by integration of the enhanced strains $\left(\boldsymbol{\varepsilon}_{\mu}^{h}=\nabla^{s}\left(\boldsymbol{u}_{\mu}^{h}\right)\right)$ defined by the operators (52-54) for the case $\overline{\mathbf{B}}=\mathbf{B}$ (that is, for a standard displacement element). Indeed, after some involved calculations, we can write

$\boldsymbol{u}_{\mu}^{h}=\boldsymbol{u}^{h}+\mathbf{M}_{\Gamma} \boldsymbol{\xi}$ in $\Omega_{e}^{h}$

for the large-scale displacements $\boldsymbol{u}^{h}=\mathbf{N d}$ (with shape functions $\left.N^{A}\left(\boldsymbol{x}^{h}\right)\right)$ and operator

$\mathbf{M}_{\Gamma}=\left[\begin{array}{llll}\mathbf{M}_{\Gamma}^{\langle 0 n\rangle} & \mathbf{M}_{\Gamma}^{\langle 0 m\rangle} & \mathbf{M}_{\Gamma}^{\langle 1 n\rangle} & \mathbf{M}_{\Gamma}^{\langle 1 m\rangle}\end{array}\right]$

given by the functions

$\mathbf{M}_{\Gamma}^{\langle 0 n\rangle}\left(\boldsymbol{x}^{h}\right)=\left(\mathcal{H}_{\Gamma_{e}^{h}}\left(\boldsymbol{x}^{h}\right)-\sum_{A \in \Omega_{e}^{h^{+}}} N^{A}\left(\boldsymbol{x}^{h}\right)\right) \boldsymbol{n}$

$\mathbf{M}_{\Gamma}^{\langle 0 m\rangle}\left(\boldsymbol{x}^{h}\right)=\left(\mathcal{H}_{\Gamma_{e}^{h}}\left(\boldsymbol{x}^{h}\right)-\sum_{A \in \Omega_{e}^{h^{+}}} N^{A}\left(\boldsymbol{x}^{h}\right)\right) \boldsymbol{m}$

$\mathbf{M}_{\Gamma}^{\langle 1 n\rangle}\left(\boldsymbol{x}^{h}\right)=\left(\mathcal{H}_{\Gamma_{e}^{h}}\left(\boldsymbol{x}^{h}\right) s-\sum_{A \in \Omega_{e}^{h^{+}}} N^{A}\left(\boldsymbol{x}^{h}\right) s^{A}\right) \boldsymbol{n}$

$+\left(\mathcal{H}_{\Gamma_{e}^{h}}\left(\boldsymbol{x}^{h}\right) r-\sum_{A \in \Omega_{e}^{h^{+}}} N^{A}\left(\boldsymbol{x}^{h}\right) r^{A}\right) \boldsymbol{m}$

$\mathbf{M}_{\Gamma}^{\langle 1 m\rangle}\left(\boldsymbol{x}^{h}\right)=\left(\mathcal{H}_{\Gamma_{e}^{h}}\left(\boldsymbol{x}^{h}\right) s-\sum_{A \in \Omega_{e}^{h^{+}}} N^{A}\left(\boldsymbol{x}^{h}\right) s^{A}\right) \boldsymbol{m}$

in terms of the two local coordinates $r=\overline{\boldsymbol{x}} \cdot \boldsymbol{n}, s=\overline{\boldsymbol{x}} \cdot \boldsymbol{m}$ and the corresponding nodal quantities $r^{A}=\overline{\boldsymbol{x}}_{A} \cdot \boldsymbol{n}$, $s^{A}=\overline{\boldsymbol{x}}_{A} \cdot \boldsymbol{m}$ of node $A$ in directions $\boldsymbol{n}$ and $\boldsymbol{m}$ of the strong discontinuity, respectively.

\section{References}

Areias PMA, Belytschko T (2006a) Two-scale shear band evolution by local partition of unity. Int J Numer Methods Eng 66:878-910

Armero F (1999) Large-scale modeling of localized dissipative mechanisms in a local continuum: applications to the numerical simulation of strain localization in rate-dependent inelastic solids. Mech Cohes Frict Mater 4:101-131

Armero F (2001) On the characterization of localized solutions in inelastic solids: an analysis of wave propagation in a softening bar. Comp Meth Appl Mech Engr 191:181-213

Armero F, Ehrlich D (2006a) Numerical modeling o f softening hinges in thin Euler-Bernoulli beams. Comput Struct 84:641-656

Armero F, Ehrlich D (2006b) Finite element methods for the multiscale modeling of softening hinge lines in plates at failure. Comp Meth Appl Mech Engr 195:1283-1324

Armero F, Garikipati K (1995) Recent advances in the analysis and numerical simulation of strain localization in inelastic solids. In: Proceedings of the 4th computational plasticity conference. CIMNE Barcelona 
Armero F, Garikipati K (1996) An analysis of strong discontinuities in multiplicative finite strain plasticity and their relation with the numerical simulation of strain localization in solids. Int J Solids Struct 33:2863-2885

Armero F, Linder C (2008) New finite elements with embedded strong discontinuities for finite deformations. Comp Meth Appl Mech Engr 197:3138-3170

Belytschko T, Black T (1999) Elastic crack growth in finite elements with minimal remeshing. Int $\mathrm{J}$ Numer Methods Eng 45:601-620

Belytschko T, Chen H, Xu J, Zi G (2003) Dynamic crack propagation based on loss of hyperbolicity and a new discontinuous enrichment. Int J Numer Methods Eng 58:1873-1905

Belytschko T, Tabbara M (1996) Dynamic fracture using element-free galerkin methods. Int $\mathrm{J}$ Numer Methods Eng 39:923-938

Bittencourt TN, Wawrzynek PA, Ingraffea AR, Sousa JL (1996) Quasi-automatic simulation of crack propagation for 2D LEFM problems. Eng Fract Mech 55:321-334

Camacho GT, Ortiz M (1996) Computational modelling of impact damage in brittle materials. Int J Solids Struc 33:2899-2938

Carter BJ, Wawrzynek PA, Ingraffea AR (2000) Automated 3 -D crack growth simulation. Int J Numer Methods Eng 47: 229-253

Dally JW (1979) Dynamic photoelastic studies of fracture. Exp Mech 19:349-361

Dolbow J, Moës N, Belytschko T (2000) Modeling fracture in Mindlin-Reissner plates with the extended finite element method. Int J Solids Struct 37:7161-7183

Duarte CA, Hamzeh ON, Liska TJ, Tworzydlo WW (2001) A generalized finite element method for the simulation of three-dimensional crack propagation. Comp Meth Appl Mech Engr 190:2227-2262

Duarte CA, Reno LG, Simone A (2007) A high-order generalized FEM for through-the-thickness branched cracks. Int J Numer Methods Eng 72:325-351

Dvorkin E, Cuitiño A, Goia G (1990) Finite elements with displacement interpolated embedded localization lines insensitive to mesh size and distortions. Int J Numer Methods Eng 30:541-564

Ehrlich D, Armero F (2005) Finite element methods for the analysis of softening plastic hinges in beams and frames. Comput Mech 35:237-264

Eshelby JD (1970) Energy relations and the energy-momentum tensor in continuum mechanics. In: Kanninen MF, Adler WF, Rosenfield AR, Jaffee RI (eds) Inelastic behaviour of solids. pp 77-115

Falk ML, Needleman A, Rice JR (2001) A critical evaluation of cohesive zone models of dynamic fracture. Journal de Physique IV 5:43-50

Fineberg J, Gross SP, Marder M, Swinney HL (1991) Instability in dynamic fracture. Phys Rev Lett 67:4

Fineberg J, Marder M (1999) Instability in dynamic fracture. Phys Rev 313:1-108

Freund LB (1998) Dynamic fracture mechanics. Cambridge University Press, Cambridge

Gao H (1993) Surface roughening and branching instabilities in dynamic fracture. J Mech Phys Solids 41:457-486

Gao H (1996) A theory of local limiting speed in dynamic fracture. J Mech Phys Solids 44:1453-1474
Gao H, Klein P (1999) Numerical simulation of crack growth in an isotropic solid with randomized internal cohesive bonds. J Mech Phys Solids 46:187-218

Hansbo A, Hansbo P (2004) A finite element method for the simulation of strong and weak discontinuities in solid mechanics. Comp Meth Appl Mech Engr 193:35233540

Huespe AE, Oliver J, Sanchez PJ, Blanco S, Sonzogni V (2006) Strong discontinuity approach in dynamic fracture simulations. Mecánica Computacional XXV:1997-2018

Hughes TJR (1987) The finite element method. Prentice-Hall, Englewood Cliffs

Ingraffea AR, Saouma V, (1985) Numerical modelling of discrete crack propagation in reinforced and plain concrete. In: Sih GC, Tomasso D (eds) Fracture mechanics of concrete: structural application and numerical calculation. pp 171-225

Kalthoff JF (1988) Shadow optical analysis of dynamic shear fracture. Opt Eng 27:835-840

Kalthoff JF (2000) Modes of dynamic shear failure in solids. Int J Fract Mech 101:1-31

Kalthoff JF, Winkler S (1987) Failure mode transition at high rates of shear loading. In: Chiem CY, Kunze H-D, Meyer LW (eds) Impact loading and dynamic behavior of materials, vol 1. DGM-Verlag, Oberursel, pp 185-195

Karedla RS, Reddy JN (2007) Modeling of crack tip high inertia zone in dynamic brittle fracture. Eng Fract Mech 74: 2084-2098

Klein PA, Foulk JW, Chen EP, Wimmer SA, Gao HJ (2001) Physics-based modeling of brittle fracture: cohesive formulation and the application of meshfree methods. Theor Appl Fract Mech 37:99-166

Knauss WG, Ravi-Chandar K (1985) Some basic problems in stress wave dominated fracture. Int J Fract Mech 27:127143

Larsson R, Steinmann P, Runesson K (1998) Finite element embedded localization band for finite strain plasticity based on a regularized strong discontinyity. Mech Cohes Frict Mater 4:171-194

Li S, Liu WK, Rosakis AJ, Belytschko T, Hao W (2002) Meshfree Galerkin simulations of dynamic shear band propagation and failure mode transition. Int J Solids Struct 39: $1213-1240$

Linder C, Armero F (2007) Finite elements with embedded strong discontinuities for the modeling of failure in solids. Int J Numer Methods Eng 72:1391-1433

Linder C, Armero F (2009) Finite elements with embedded branching Finite. Elem Anal Des 45:280-293

Manzoli OL, Shing PB (2006) A general technique to embed non-uniform discontinuities into standard solid finite elements. Comput Struct 84:742-757

Mason JJ, Rosakis AJ, Ravichandran G (1994) Full field measurements of the dynamic deformaiton field around a growing adiabatic shear band at the tip of a dynamically loaded crack or notch. J Mech Phys Solids 42:1679-1697

Marusich TD, Ortiz M (1995) Modelling and simulation of high-speed machining. Int $\mathrm{J}$ Numer Methods Eng 38: 3675-3694

Medyanik SN, Liu WK, Li S (2007) On criteria for dynamic adiabatic shear band propagation. J Mech Phys Solids 55:1439-1461 
Moës N, Dolbow J, Belytschko T (1999) A finite element method for crack growth without remeshing. Int J Numer Methods Eng 46:131-150

Needleman A (1987) A continuum model for void nucleation by inclusion debonding. J Appl Mech 54:525-531

Needleman A, Tvergaard V (1994a) Mesh effects in the analysis of dynamic ductile crack growth. Eng Fract Mech 47:75-91

Needleman A, Tvergaard V (1994b) Analysis of brittle-ductile transition under dynamic shear loading. Int J Solids Struct 32:2571-2590

Oliver J (1996a) Modelling strong discontinuities in solid mechanics via strain softening constitutive equations. Parts 1: fundamentals. Int J Numer Methods Eng 39:3575-3600

Oliver J (1996b) Modelling strong discontinuities in solid mechanics via strain softening constitutive equations. Part 2: numerical simulation. Int $\mathbf{J}$ Numer Methods Eng 39:3601-3623

Oliver J, Huespe AE, Sanchez PJ (2006) A comparitive study on finite elements for capturing strong discontinuities: E-FEM vs. X-FEM. Comp Meth Appl Mech Engr 195:4732-4752

Ortiz M, Pandolfi A (1999) Finite deformation irreversible cohesive elements for three-dimensional crack-propagation analysis. Int J Numer Methods Eng 44:1267-1282

Ortiz M, Quigley JJ (1991) Adaptive mesh refinement in strain localization problems. Comp Meth Appl Mech Engr 90:781-804

Rabczuk T, Samaniego E (2008) Discontinuous modelling of shear bands using adaptive meshfree methods. Comp Meth Appl Mech Engr 197:641-658

Ramulu M, Kobayashi AS (1985) Mechanics of crack curving and branching - a dynamic fracture analysis. Int J Fract Mech 27:187-201

Ravi-Chandar K, Knauss WG (1984a) An experimental investigation into dynamic fracture: II. Microstructural aspects. Int J Fract Mech 26:65-80

Ravi-Chandar K, Knauss WG (1984) An experimental investigation into dynamic fracture: III on steady-state crack propagation and crack branching. Int J Fract Mech 26:141-154

Ravi-Chandar K (1995) On the failure mode transitions in polycarbonate under dynamic mixed-mode loading. J Mech Phys Solids 26:925-938

Remmers JJC, de Borst R, Needleman A (2008) The simulation of dynamic crack propagation using the cohesive segment method. J Mech Phys Solids 56:70-92

Satoh H (1996) On the crack propagation in brittle materials. Thesis, Department of Aeronautics and Astronautic, University of Tokyo, Japan
Sharon E, Fineberg J (1996) Microbranching instability and the dynamic fracture of brittle materials. Phys Rev B Condens Matter 54:7128-7139

Sharon E, Fineberg J (1999) Confirming the continuum theory of dynamic brittle fracture for fast cracks. Nature 397: 333-335

Simo JC, Oliver J, Armero F (1993) An analysis of strong discontinuities induced by strain-softening in rate independent inelastic solids. Comput Mech 12:277-296

Song J-H, Areias PMA, Belytschko T (2006) A method for dynamic crack and shear band propagation with phanotom nodes. Int J Numer Methods Eng 67:868-893

Tvergaard V (1990) Effect of fibre debonding in a whisker-reinforced metal. Mater Sci Eng A 125:203-213

Wells GN, Sluys LJ (2001) A new method for modelling cohesive cracks using finite elements. Int J Numer Methods Eng 50:2667-2682

Wells GN, Sluys LJ, Borst R (2002) Simulating the propagation of displacement discontinuities in a regularized strain-softening medium. Int J Numer Methods Eng 53:1235-1256

de Xu XP, Needleman A (1994) Numerical simulations of fast crack growth in brittle solids. J Mech Phys Solids 42: 1397-1434

Yoffe EH (1951) The moving Griffith crack. Philos Mag 42: 739-750

Zhang Z, Paulino GH, Celes W (2007) Extrinsic cohesive modelling of dynamic fracture and microbranching instability in brittle materials. Int J Numer Methods Eng 72:893-923

Zhou M, Rosakis AJ, Ravichandran G (1996a) Dynamically propagating shear bands in impact-loaded prenotched plates-I. Experimental investigations of temperature signatures and propagation speed. J Mech Phys Solids 44: 981-1006

Zhou M, Ravichandran G, Rosakis AJ (1996b) Dynamically propagating shear bands in impact-loaded prenotched plates-II numerical simulations. J Mech Phys Solids 44:1007-1032

Zhou F, Molinari JF (2004) Dynamic crack propagation with cohesive elements: a methodology to address mesh dependency. Int J Numer Methods Eng 59:1-24

Zhou F, Molinari JF, Shioya T (2005) A rate-dependent cohesive model for simulating dynamic crack propagation in brittle materials. Eng Fract Mech 72:1383-1410

Zi G, Rabczuk T, Wall W (2007) Extended meshfree methods without branch enrichment for cohesive cracks. Comput Mech 40:367-382 